\title{
Post-Collision Interaction with Wannier electrons
}

\author{
M. Yu. Kuchiev $\ddagger$ \\ School of Physics, The University of New South Wales, Sydney 2052, Australia
}

\begin{abstract}
A theory of the Post-Collision Interaction (PCI) is developed for the case when an electron atom impact results in creation of two low-energy Wannier electrons and an ion excited into an autoionizing state. The following autoionization decay exposes the Wannier pair to the influence of PCI resulting in variation of the shape of the line in the autoionization spectrum. An explicit dependence of the autoionization profile on the wave function of the Wannier pair is found. PCI provides an opportunity to study this wave function for a wide area of distances.
\end{abstract}

PACS numbers: $34.80 . \mathrm{Dp}$

Short title: PCI with Wannier electrons

October 30, 2018

$\ddagger$ E-mail: kuchiev@newt.phys.unsw.edu.au 


\section{Introduction}

This paper develops a theoretical approach allowing one to consider PCI in the Wannier regime. The interest in this problem has been initiated by recent experiment [1] followed by theoretical work [2] in which the electron impact in the vicinity of the atomic inner shell ionization threshold

$$
\mathrm{e}+\mathrm{A} \rightarrow \mathrm{e}_{\alpha}+\mathrm{e}_{\beta}+\mathrm{A}^{+*}
$$

was studied. In this reaction the inelastically scattered electron $\mathrm{e}_{\alpha}$ and the knocked out electron $\mathrm{e}_{\beta}$ have low energy propagating in the Coulomb field of the positive ion $\mathrm{A}^{+*}$. These low-energy electrons are known as the Wannier pair. The Coulomb interaction of Wannier electrons between themselves as well as with the field of the ion results in the Wannier power law [3] for the cross-section

$$
\sigma \sim \varepsilon^{\mu}
$$

where $\varepsilon$ is the energy excess over the ionization threshold and the exponent $\mu$ depends non-trivially on the ion charge $Z$

$$
\mu=\frac{1}{4}\left[\left(\frac{100 Z-9}{4 Z-1}\right)^{1 / 2}-1\right] .
$$

A distinctive property of reaction (1.1) is the finite life-time of the inner-shell vacancy in the ion $\mathrm{A}^{+*}$. Its decay

$$
\mathrm{e}_{\alpha}+\mathrm{e}_{\beta}+\mathrm{A}^{+*} \rightarrow \mathrm{e}_{\alpha}+\mathrm{e}_{\beta}+\mathrm{e}_{\gamma}+\mathrm{A}^{2+}
$$

creates the fast Auger electron $\mathrm{e}_{\gamma}$ and doubly-charged ion $\mathrm{A}^{2+}$. The fact that the pair $\mathrm{e}_{\alpha}, \mathrm{e}_{\beta}$ has low energy guarantees that at the moment of the decay it is situated in some close vicinity of the ion. Therefore the pair should strongly interact with the products of the Auger decay, i.e. the doubly charged ion and Auger electron. An interaction of this type is known in literature as the Post-Collision Interaction (PCI). What distinguishes reaction (1.1),(1.4) is the fact that the Wannier pair takes part in PCI. Previously PCI was extensively studied in the near threshold region for the case when a single low-energy electron is localized in the vicinity of the atom at the moment of the autoionization decay.

Thus reaction (1.1), (1.4) combines the two problems of the final state interaction, the Wannier problem and PCI. Previously they both had been extensively studied experimentally as well as theoretically but in those situations when only one of them, either the Wannier problem or PCI had manifested itself in the final state.

The main purpose of this paper is development of the theory describing PCI in reaction (1.1), (1.4) as well as in similar situations in electron or atomic collisions or in photoionization. One of the interesting qualitative questions arising from the reaction 
(1.1), (1.4) is a possible influence of PCI on the Wannier power law (1.2). In the field of the singly charged ion the Wannier exponent (1.3) is $\mu_{1} \simeq 1.127$, while in the field of the doubly charged ion it is $\mu_{2} \simeq 1.056$. If the energy of the Auger electron $\mathrm{e}_{\gamma}$ is high enough then it does not interact strongly with the pair $\mathrm{e}_{\alpha}, \mathrm{e}_{\beta}$ and the decay could be considered as a switch of the ion charge from the intermediate-state value $Z=1$ to the final-state value $Z=2$. The question is how this charge variation affects the exponent. We will demonstrate that for sufficiently low above-threshold energy the exponent is equal to $\mu_{2}$, while for higher energies it is $\mu_{1}$. This fact agrees with the following simple physical picture. When above-threshold energy is very low then the Wannier pair is located so close to the ion at the moment of the decay that its separation is negligible compared with an important in the problem radius of the Coulomb zone. Therefore one can neglect this separation, neglecting as well the very fact of existence of the intermediate-state resonance. Thus in this case the pair is influenced by the field of the final-state ion with the charge $Z=2$. In contrast for high above-threshold energy the Wannier pair is located well outside the radius of the Coulomb zone at the moment of the Auger decay and therefore is not strongly influenced by the field of the doubly-charged ion. The main characteristics of the pair in this case are governed by the ion in the intermediate state with $Z=1$.

The paper is organized as follows. Section 2 gives a short introduction to the Wannier problem which was theoretically studied in a number of papers. The variety of approaches developed and the comprehensive list of references can be found in [4] [8]. Section 3 presents a short review of essential ideas of PCI. The literature on the theory of PCI can be found in [9]. These introductory notes permit presenting basic ideas of the two problems from a particular point of view which provides the easiest way for further development in Sections 4 and 5 where a way is found to describe PCI when Wannier electrons are involved.

Generally speaking the problem considered looks very complicated being dependent on the Coulomb interaction of three electrons and the ion. There are, however, important simplifications. Firstly, we assume that the Wannier pair possesses low energy, while the Auger electron is so fast that one can neglect its interaction with the pair. This allows us to reformulate the problem in terms of the Wannier pair only. Still it is a dynamical three-body problem. Another important simplification comes from the cornerstone of the Wannier problem [3]. In order to escape from the ionic field Wannier electrons should move along a particular classical trajectory. This motion can be considered as a collective mode describing essential properties of the system. One can easily verify, see Section 2, that this mode can be described in terms of an effective single-particle Coulomb problem. Of course there are important corrections arising from the threebody nature of the problem and making it so specific and interesting. The point is that knowing the reliable initial approximation one can find these corrections in a clear 
analytical form.

These arguments show that one should expect the problem considered to have a clear solution. Finding and describing it in simple physical terms is the major goal of this paper. The main result obtained is the formula which presents the autoionization profile $R_{\text {pci }}$ distorted by PCI in the form

$$
R_{\mathrm{pci}}=K_{\mathrm{w}} R_{\mathrm{c}}
$$

The factor $R_{\mathrm{c}}$ here arises from the most simple approximation for the Wannier problem described by the above mentioned effective single-particle Coulomb problem. Due to this reason we will call it the Coulomb factor. This factor depends on the overlapping integral of the Coulomb wave functions. It is interesting that a similar factor describes PCI in the traditional situation when there is only one low-energy electron. The only distinction is that in the later case there is a true single-particle Coulomb problem, while for the Wannier pair the Coulomb problem arises as an effective approximation. Still the similarity is so close that one can consider the Coulomb factor $R_{\mathrm{c}}$ as a well known quantity which was well studied previously both experimentally and theoretically. In contrast the factor $K_{\mathrm{w}}$ takes into account more subtle and interesting properties of the process which are essential for PCI with the Wannier pair and have no analogy in PCI with one low-energy electron. Due to this reason we will call it the Wannier factor. In Section 5 an explicit analytical formula for this factor is derived. The Wannier factor found depends on the normalization coefficient of the Wannier electrons wave function. It is interesting that the Wannier power law (1.2) arises from the properties of the same coefficient considered in the region of small separations of the pair from the atom. In contrast, when PCI is studied this coefficient manifests itself in a wide range of distances. This property of the profile (1.5) opens a possibility for experimental studies

of the normalization coefficient of the Wannier pair wave function in a wide area of distances.

The results obtained in Sections 4,5 are illustrated in Section 6 for the electron impact near-threshold K-shell excitation of Ne. Section 7 presents a short description of the major results.

\section{Wannier problem}

Consider two low-energy electrons in the field of an ion with the charge $Z$. The Coulomb repulsion between the electrons makes opposite directions the most favorable for them [3]. To simplify the following consideration let us use this fact developing the following model. Let us assume that the electrons are moving in opposite directions neglecting fluctuations in their angular distribution. Moreover, let us neglect as well the orbital moment. Thus in this model angular variables are neglected which makes it essentially 
one-dimensional. This model was applied earlier for description of the Wannier power law [10]. It is important that the most interesting part of the Wannier problem, its instability against a non-symmetrical distribution of energy between the two electrons, is taken into account in full. Moreover, we will argue in Section 7 that our final results do not depend on model assumptions. One of the main advantages of the model is simplification of notation, which inevitably is to be sufficiently complicated.

Our strategy in this Section is to first consider the classical approximation and then develop the quantum picture using the semiclassical approach which gives a correct description of the process for low above-threshold energy. The semiclassical approach to the Wannier problem was developed in detail in [4 to describe the near-threshold behaviour of the reaction (1.1). In this case one is mainly interested in the behaviour of the wave function of the Wannier pair for small separations. We will see that to describe PCI it is necessary to know the wave function for any separation, not necessarily in the vicinity of the atom. The convenient representation for this wave function valid for an arbitrary separation is given below in equation (2.62) which is the main result of this Section.

\subsection{Classical trajectories}

Consider classically the problem of two electrons $\mathrm{e}_{\alpha}, \mathrm{e}_{\beta}$ in the framework of the aboveformulated one-dimensional model in which electrons are supposed to move in opposite directions with zero orbital moments. Such a system is characterized by two radii $r_{\alpha}, r_{\beta} \geq 0$. The Lagrangian in these coordinates is

$$
L\left(r_{\alpha}, r_{\beta}, \dot{r}_{\alpha}, \dot{r}_{\beta}\right)=\frac{\dot{r}_{\alpha}^{2}}{2}+\frac{\dot{r}_{\beta}^{2}}{2}+\frac{Z}{r_{\alpha}}+\frac{Z}{r_{\beta}}-\frac{1}{r_{\alpha}+r_{\beta}},
$$

where $Z$ is the charge of the ion. It is convenient to introduce hyper-spherical coordinates

$$
\left\{\begin{array}{l}
r_{\alpha}=r \cos \left(\chi+\frac{\pi}{4}\right) \\
r_{\beta}=r \cos \left(\chi-\frac{\pi}{4}\right)
\end{array}\right.
$$

in which $r \geq 0$ describes the overall distance of the pair from the ion, and $\chi,-\pi / 4 \leq$ $\chi \leq \pi / 4$ shows how strongly the pair is deviated from the symmetrical configuration $r_{\alpha}=r_{\beta}$, in which $\chi=0$. In these coordinates the Lagrangian is

$$
L(r, \chi, \dot{r}, \dot{\chi})=\frac{\dot{r}^{2}}{2}+\frac{r^{2} \dot{\chi}^{2}}{2}-U(r, \chi) .
$$

The potential energy

$$
U(r, \chi)=-\frac{w(\chi)}{r}
$$


can be looked at as a Coulomb field produced by a $\chi$-dependent charge

$$
w(\chi)=\frac{(2 Z-1) \cos 2 \chi+2 Z}{\sqrt{2} \cos \chi \cos 2 \chi} \geq 0 .
$$

The function $w(\chi)$ has a minimum for the symmetrical configuration $\chi=0$. It manifests itself as a maximum at $\chi=0$ of the potential $U(r, \chi)$ considered as a function of $\chi$. This property of the potential is known as the Wannier ridge. The ridge makes the classical system unstable. The instability results in the most important property of the Wannier problem. In order to escape from the Coulomb field of the ion the two electrons should propagate in the vicinity of the symmetrical configuration. Otherwise one of them would be trapped by the ion field, i.e. the pair fall off the ridge. That is why the motion in the vicinity of the top of the ridge, where the pair is stable plays such an important role and is considered in detail below.

In the vicinity of $\chi=0$ the function $w(\chi)$ can be expanded in the Taylor series

$$
w(\chi) \approx w+\frac{w^{\prime \prime}}{2} \chi^{2}
$$

where the coefficients are

$$
\begin{aligned}
& w=\frac{4 Z-1}{\sqrt{2}}, \\
& w^{\prime \prime}=\frac{12 Z-1}{\sqrt{2}} .
\end{aligned}
$$

Equation (2.3) results in equations of motion

$$
\begin{aligned}
& \ddot{r}=r \dot{\chi}^{2}-\frac{w(\chi)}{r^{2}}, \\
& \frac{d}{d t}\left(r^{2} \dot{\chi}\right)=\frac{1}{r} \frac{d w}{d \chi}(\chi) .
\end{aligned}
$$

The trajectory along the top of the Wannier ridge satisfies

$$
\begin{aligned}
& \chi_{0}=0, \\
& \ddot{r}_{0}=-\frac{w}{r_{0}^{2}} .
\end{aligned}
$$

The second equation coincides with the equation describing the motion of a single particle in the field of the Coulomb charge $w$. We will refer to this motion, which plays an important role below, as an effective single-particle Coulomb problem. The first integral of (2.12)

$$
\frac{\dot{r}_{0}^{2}}{2}-\frac{w}{r_{0}}=\epsilon
$$

describes the energy conservation law. Using it one finds the ridge-top trajectory

$$
t=\int \frac{d r_{0}}{\left.\sqrt{2\left(\epsilon+w / r_{0}\right.}\right)}=r_{0}(t)\left[2\left(\epsilon+\frac{w}{r_{0}(t)}\right)\right]^{1 / 2}
$$




$$
+\frac{w}{\sqrt{2 \epsilon}} \ln \left\{1+\frac{2 \epsilon r_{0}(t)}{w}\left[1+\left(1+\frac{w}{\epsilon r_{0}(t)}\right)^{1 / 2}\right]\right\} .
$$

For small deviations from the ridge-top trajectory one obtains the linear equation for $\chi=\delta \chi$ using expansion (2.6)

$$
r \frac{d}{d t}\left(r^{2} \dot{\chi}\right)=w^{\prime \prime} \chi
$$

To simplify notation the ridge-top trajectory is denoted here by $r=r_{0}(t)$ defined in (2.14). For further applications we need to consider solutions of (2.15) in detail. It is convenient to change the argument $t \rightarrow x$ in such a way that

$$
r^{2} \frac{d}{d t}=-\frac{w}{2 \sqrt{2 \epsilon}} \frac{d}{d x} .
$$

This is achieved for

$$
x=\frac{1}{2}\left[\left(1+\frac{w}{\epsilon r}\right)^{1 / 2}+1\right] .
$$

The new variable $x=x(\epsilon r)$ satisfies

$$
x \rightarrow \begin{cases}\frac{1}{2}\left(\frac{w}{\epsilon r}\right)^{1 / 2} & \epsilon r \ll 1 \\ 1+\frac{w}{4 \epsilon r} & \epsilon r \gg 1\end{cases}
$$

Using (2.16) one can rewrite (2.15) as

$$
\frac{d^{2} \chi(x)}{d x^{2}}=\frac{\lambda}{x(x-1)} \chi(x),
$$

where $\lambda$ is the coefficient

$$
\lambda=2 \frac{w^{\prime \prime}}{w}=2 \frac{12 Z-1}{4 Z-1} .
$$

Scaling the function

$$
\chi(x) \rightarrow y(x)=\chi(x) / x
$$

we find that the motion in the vicinity of the top of the ridge is described by the following linear differential equation

$$
x(1-x) y^{\prime \prime}(x)+2(1-x) y^{\prime}(x)+\lambda y(x)=0,
$$

in which one recognizes the known [1] hypergeometric equation whose parameters $\alpha, \beta, \gamma$

$$
\alpha=\nu+1, \quad \beta=-\nu, \quad \gamma=2
$$

are restricted by the condition on $\nu$

$$
\nu(\nu+1)=\lambda .
$$


Solving this equation and using (2.19) one finds an explicit form for this parameter

$$
\nu=\frac{1}{2}\left[\left(\frac{100 Z-9}{4 Z-1}\right)^{1 / 2}-1\right],
$$

which governs the Wannier problem, compare (2.24) with (1.3).

It is convenient to choose a set of basic solutions $y_{1}(x), y_{2}(x)$ of $(2.21)$ as

$$
\begin{aligned}
& y_{1}=(x-1)^{\gamma-\alpha-\beta} F(\gamma-\alpha, \gamma-\beta, \gamma+1-\alpha-\beta, 1-x), \\
& y_{2}=x^{-\alpha} F(\alpha, \alpha+1-\gamma, \alpha+1-\beta, 1 / x),
\end{aligned}
$$

where $F(\alpha, \beta, \gamma, x)$ is the hypergeometric function. Then an arbitrary solution of (2.18) can be presented as a linear combination

$$
\chi(x)=a_{1} f(x)+a_{2} g(x)
$$

of functions $f(x)=x y_{1}(x), g(x)=x y_{2}(x)$ having the following form

$$
\begin{aligned}
& f(x)=x(x-1) F(-\nu+1, \nu+2,2,1-x) \\
& g(x)=\zeta \frac{\nu+1}{2 \nu+1} x^{-\nu} F\left(\nu, \nu+1,2 \nu+2, \frac{1}{x}\right) .
\end{aligned}
$$

To simplify the subsequent formulae a normalization coefficient $\zeta$ in $(2.27)$ is chosen to be

$$
\zeta=\frac{[\Gamma(\nu+1)]^{2}}{\Gamma(2 \nu+1)}
$$

Here $\Gamma(x)$ is the usual Euler gamma function. We will need to know the asymptotic conditions for the functions $f(x), g(x)$, which are easily found from $(2.26),(2.27)$

$$
\begin{gathered}
f(x) \rightarrow\left\{\begin{array}{cc}
\frac{1}{\zeta(\nu+1)} x^{\nu+1} & x \rightarrow \infty \\
x-1 & x \rightarrow 1
\end{array}\right. \\
g(x) \rightarrow\left\{\begin{array}{cl}
\zeta \frac{\nu+1}{2 \nu+1} x^{-\nu} & x \rightarrow \infty \\
1 & x \rightarrow 1 .
\end{array}\right.
\end{gathered}
$$

At the end points $x=1$ and $x=\infty$ one of the functions, either $f(x)$ or $g(x)$, is singular while the other one is regular making them convenient for applications. Notice that the derivative $g^{\prime}(x)$ remains singular at $x \rightarrow 1$

$$
g^{\prime}(x) \rightarrow 2 \frac{w^{\prime \prime}}{w}[\ln (x-1)+d]+O[(x-1) \ln (x-1)] .
$$

Here $d$ is a constant

$$
d=2[\psi(\nu+1)+C]
$$


where $\psi(x)=\Gamma^{\prime}(x) / \Gamma(x)$ and $C=-\psi(1) \simeq 0.577$ is the Euler constant. One more necessary parameter in the problem is the Wronskian of the functions $f(x), g(x)$ which can be found from asymptotic conditions (2.29),(2.30)

$$
f^{\prime}(x) g(x)-f(x) g^{\prime}(x)=1 .
$$

The given consideration describes in detail the main trajectory along the top of the Wannier ridge (2.12) as well as small deviations from it given in (2.25), $(2.26,(2.27)$ in which the variable $x=x(\epsilon r)$ is defined in (2.16), (2.14).

\subsection{Quantum description}

Essential to the problem distances are in the Coulomb zone

$$
r \sim \frac{1}{\epsilon} \equiv r_{\mathrm{c}} .
$$

In this region the Coulomb force is $F_{\mathrm{c}} \sim 1 / r^{2} \sim \epsilon^{2}$. The variation of the Coulomb energy on the wavelength $(1 / \sqrt{\epsilon}) F_{\mathrm{c}} \sim \epsilon^{3 / 2}$ proves to be smaller than the kinetic energy $\sim \epsilon$ provided the energy is low

$$
\epsilon \ll 1 \text {. }
$$

It is supposed in this paper that the later condition is valid. The given estimations show that in this case the semiclassical approximation turns out to be correct permitting one to write the wave function in the well known form

$$
\psi_{P}(q)=N(q, P) \exp [i S(q, P)],
$$

where $S(q, P)$ is the classical action. The wave function depends on a set of coordinates $q$ and quantum numbers $P$, the later ones play a role of parameters. It is convenient to choose $P$ as momenta conjugate to coordinates $q$. To be specific consider the convergent wave function for the final state $\psi=\psi^{(-)}$which is the most interesting in the Wannier problem. This wave function may be looked at as a transition amplitude from the state in which coordinates $q$ of the Wannier pair are in the vicinity of the atom to the final state in which momenta $P$ describe the motion at an infinite separation. In classical terms the transition should be described by a trajectory with initial coordinates $q$ and final momenta $P$. These two sets of parameters $q$ and $P$ fix the trajectory. The classical action depends on both these sets of variables $S=S(q, P)$. It is well known that the non-exponential factor $N(q, P)$ in the wave function depends on the derivatives of the action [12]

$$
N(q, P)=\left[\operatorname{det}\left(-\frac{\partial^{2} S}{\partial q_{i} \partial P_{j}}\right)\right]^{1 / 2} .
$$


Let us choose a set of coordinates $q$ as $q=(r, \eta)$, where $\eta$ is the momentum conjugate to the variable $\chi$ introduced in (2.2)

$$
\eta=\frac{\partial L}{\partial \dot{\chi}}=r^{2} \dot{\chi}
$$

A convenience of this choice becomes apparent soon. The corresponding momenta are $P=(\bar{p}, \bar{\chi})$. (More accurately the momentum conjugate to $\eta$ should be written as $-\chi$. The minus sign is dropped to simplify notation.) Here $p$ is a momentum conjugate to $r$

$$
p=\frac{\partial L}{\partial \dot{r}}=\dot{r}
$$

To avoid confusion the bar symbol is used to mark the variables belonging to the final point of the trajectory for infinite separations. In this notation the energy of the pair is written as $\epsilon=\bar{p}^{2} / 2$, and the action looks like $S=S(r, \eta ; \bar{p}, \bar{\chi})$. Calculating the action we should consider the region in the vicinity of the Wannier ridge where both $\bar{\chi}$ and $\eta$ are small. Therefore we can expand the action in powers of these variables

$$
S(r, \eta ; \bar{p}, \bar{\chi})=\hat{S}_{0}(r, \bar{p})+\hat{\Sigma}(r, \eta ; \bar{p}, \bar{\chi}),
$$

where

$$
\hat{S}_{0}(r, \bar{p})=S(r, 0 ; \bar{p}, 0)=\int_{r}^{\bar{r}} \sqrt{\bar{p}^{2}+2 \frac{w}{r^{\prime}}} d r^{\prime}-\bar{p} \bar{r}
$$

describes a one-dimensional motion along the ridge-top trajectory governed by the effective Coulomb charge $w$. We will recognize later, see (2.55), that a slightly different choice of the effective charge proves to be more convenient. The quantities which will be recognized be affected by this redefinition are marked by the hat symbol. The quantity $\hat{\Sigma}(r, \eta ; \bar{p}, \bar{\chi})$ depends on the derivatives of $S$. The first derivatives for the ridge-top trajectory are obviously zero. Therefore, the expansion starts from the second powers of $\bar{\chi}, \eta$. It is shown below, see discussion after (2.62), that it is sufficient to restrict our consideration to these lowest order terms writing

$$
\hat{\Sigma}(r, \eta ; \bar{p}, \bar{\chi})=\frac{\bar{\chi}^{2}}{2 \bar{p}} \hat{A}+\bar{\chi} \eta B+\frac{\eta^{2} \bar{p}}{2} C .
$$

The factors $1 / \bar{p}$ and $\bar{p}$ are included here to simplify the subsequent formulae. The coefficients of this expansion are functions of $r$ and $\bar{p}$. In order to find them let us remember that $\chi$ and $\eta$ are the conjugate variables and therefore

$$
\begin{aligned}
& \frac{1}{\bar{p}} \hat{A}=\frac{\partial^{2} S}{\partial \bar{\chi}^{2}}=\left(\frac{\partial \bar{\eta}}{\partial \bar{\chi}}\right)_{\eta}, \\
& B=\frac{\partial^{2} S}{\partial \bar{\chi} \partial \eta}=\left(\frac{\partial \bar{\eta}}{\partial \eta}\right)_{\bar{\chi}},
\end{aligned}
$$




$$
\bar{p} C=\frac{\partial^{2} S}{\partial \eta^{2}}=\left(\frac{\partial \chi}{\partial \eta}\right)_{\bar{\chi}} .
$$

The derivatives in (2.43), 2.44), 2.45) describe the variation of the coordinate and momentum under variation of the trajectory. This variation in turn is described by variation of the coefficients $a_{1}, a_{2}$ in (2.25) which governs the coordinate $\chi(x)=$ $a_{1} f(x)+a_{2} g(x)$ as well as the momentum $\eta$ conjugate to $\chi$

$$
\eta=r^{2} \dot{\chi}=-\frac{w}{2 \bar{p}} \chi^{\prime}(x)=-\frac{w}{2 \bar{p}}\left(a_{1} f^{\prime}(x)+a_{2} g^{\prime}(x)\right) .
$$

Using (2.25),(2.46) one finds from (2.43)

$$
\hat{A}=-\frac{w}{2}\left[\frac{\left(d a_{1}\right) \bar{f}^{\prime}+\left(d a_{2}\right) \bar{g}^{\prime}}{\left(d a_{1}\right) \bar{f}+\left(d a_{2}\right) \bar{g}}\right]_{\eta}
$$

where according to (2.46) the condition $\eta=$ const restricts variations of coefficients $d a_{1}, d a_{2}$

$$
\left(d a_{1}\right) f^{\prime}+\left(d a_{2}\right) g^{\prime}=0 .
$$

Combining the last two equations we find

$$
\hat{A}=\frac{w}{2} \frac{\bar{f}^{\prime} g^{\prime}-f^{\prime} \bar{g}^{\prime}}{f^{\prime} \bar{g}-\bar{f} g^{\prime}} .
$$

The other derivatives are calculated similarly

$$
\begin{aligned}
& B=\frac{f^{\prime} g-f g^{\prime}}{f^{\prime} \bar{g}-\bar{f} g^{\prime}}=\frac{1}{f^{\prime} \bar{g}-\bar{f} g^{\prime}}, \\
& C=-\frac{2}{w} \frac{f \bar{g}-\bar{f} g}{f^{\prime} \bar{g}-\bar{f} g^{\prime}} .
\end{aligned}
$$

Evaluating the last identity in (2.48) the Wronskian (2.33) was used. All three coefficients turn out to be functions of the single variable $x, A=A(x), B=B(x), C=$ $C(x)$. This property of the coefficients was anticipated when normalization factors $1 / \bar{p}$ and $\bar{p}$ were introduced in their definitions in (2.42).

We can now use the fact that for the final state $\bar{r}=\infty$, and therefore according to (2.17) $\bar{x}=1$. From $(2.29),(2.30)$ we find $\bar{f}=0, \quad \bar{f}^{\prime}=1, \quad \bar{g}=1$, resulting in

$$
\begin{aligned}
& \hat{A}(x)=\frac{w}{2}\left(\frac{g^{\prime}(x)}{f^{\prime}(x)}-\bar{g}^{\prime}\right), \\
& B(x)=\frac{1}{f^{\prime}(x)} \\
& C(x)=-\frac{2}{w} \frac{f(x)}{f^{\prime}(x)} .
\end{aligned}
$$


All these quantities are well defined functions of $x$, except for the constant $\bar{g}^{\prime}$ which according to (2.31) is logarithmically divergent $\bar{g}^{\prime}=g^{\prime}(\bar{x}) \sim \ln (\bar{x}-1), \quad \bar{x} \rightarrow \infty$. This divergence manifests itself in the action as a term

$$
-\frac{w \bar{\chi}^{2}}{4 \bar{p}} g^{\prime}(\bar{x})=\frac{w^{\prime \prime} \bar{\chi}^{2}}{2 \bar{p}}\left[\ln \left(\frac{2 \bar{p}^{2} \bar{r}}{w}\right)-d\right] .
$$

In order to deal with this problem let us remember that the Coulomb action $\hat{S}_{0}(2.41)$ reveals the logarithmic divergence as well, $\hat{S}_{0} \sim(w / \bar{p}) \ln \left(2 \bar{p}^{2} \bar{r} / w\right), \quad \bar{r} \rightarrow \infty$. This later divergence proves harmless. Really, it takes place for an effective one-dimensional Coulomb problem described by the only coordinate $r$. For this simple situation the Coulomb logarithmic divergence is of course well studied and cured by textbook methods, see [1]. Having this in mind let us "renormalize" $\hat{S}_{0}$ and $\hat{\Sigma}, \hat{S}_{0} \rightarrow S_{0}, \hat{\Sigma} \rightarrow \Sigma$

$$
S(r, \eta ; \bar{p}, \bar{\chi})=S_{0}(r, \bar{p})+\Sigma(r, \eta ; \bar{p}, \bar{\chi})
$$

in a way that makes the divergence (2.53) apparently harmless as well. The action $S_{0}(r, \bar{p})$ in (2.54) is defined as

$$
S_{0}(r, \bar{p})=\int_{r}^{\bar{r}} \sqrt{\bar{p}^{2}+2 \frac{w(\bar{\chi})}{r^{\prime}}} d r^{\prime}-\bar{p} \bar{r} .
$$

It describes the one-dimensional motion in the field of the Coulomb charge $w(\bar{\chi})$ (2.5) which explicitly depends on $\bar{\chi}^{2}$, see (2.6). This property distinguishes it from the "nonrenormalized" quantity $\hat{S}_{0}$ (2.41) which does not depend on $\bar{\chi}$. The renormalized $\Sigma$

$$
\Sigma(r, \eta ; \bar{p}, \bar{\chi})=\hat{\Sigma}(r, \eta ; \bar{p}, \bar{\chi})-\frac{w^{\prime \prime} \bar{\chi}^{2}}{2} \int_{r}^{\bar{r}} \frac{d r^{\prime}}{r^{\prime} \sqrt{\bar{p}^{2}+2 w / r^{\prime}}}
$$

is defined in such a way that it compensates for the dependence of $S_{0}$ on $\bar{\chi}^{2}$. Using these new definitions we find

$$
\Sigma(r, \eta ; \bar{p}, \bar{\chi})=\frac{\bar{\chi}^{2}}{2 \bar{p}} A(x)+\bar{\chi} \eta B(x)+\frac{\eta^{2} \bar{p}}{2} C(x),
$$

where $B(x), C(x)$ remain equal to definitions given in $(2.51),(2.52)$. In contrast $A(x)$ differs from the "non-renormalized" value $\hat{A}(x)$ (2.50), namely

$$
A(x)=\frac{w}{2} \frac{g^{\prime}(x)}{f^{\prime}(x)}+w^{\prime \prime} h(x) .
$$

Here $h(x)$ is a new function whose definition follows from (2.53), 2.56)

$$
h(x)=-\bar{p} \int_{r}^{\bar{r}} \frac{d r^{\prime}}{r^{\prime} \sqrt{\bar{p}^{2}+2 w / r^{\prime}}}+\ln \left(\frac{2 \bar{p}^{2} \bar{r}}{w}\right)-d .
$$


Calculating the integral and taking the limit $\bar{r} \rightarrow \infty$ we find

$$
h(x)=\ln \left[\frac{r}{w}\left(\bar{p}^{2}+\frac{w}{r}+\bar{p} \sqrt{\bar{p}^{2}+\frac{2 w}{r}}\right)\right]-d=\ln \frac{x}{x-1}-d,
$$

where $d$ was defined in (2.32).

The non-exponential factor $N(q, P)$ in the wave function (2.36) is easily calculated because with the necessary accuracy it is

$$
N(r, \eta ; \bar{p}, \bar{\chi}) \simeq N_{0}(r, \bar{p})\left(\frac{\partial^{2} S}{\partial \bar{\chi} \partial \bar{\eta}}\right)^{1 / 2}=N_{0}(r, \bar{p}) B(x) .
$$

Here the first factor $N_{0}(r, \bar{p})$ is related to the effective one-dimensional Coulomb problem

$$
N_{0}(r, \bar{p})=\left(-\frac{\partial^{2} S_{0}}{\partial r \partial \bar{p}}\right)^{1 / 2}
$$

The factor $B(x)$ was calculated previously in (2.51).

Combining the above calculated quantities we can present the wave function (2.36) in the following final form

$$
\psi_{p, \chi}(r, \eta)=\sqrt{B(x)} \exp [i \Sigma(r, \eta ; p, \chi)] \phi_{p}^{(-)}(r ; w(\chi)) .
$$

We need no more to distinguish by a bar symbol the variables at the final point supposing that $p \equiv \bar{p}, \chi \equiv \bar{\chi}$. Evaluating (2.62) it was taken into account that $N_{0}(r) \exp \left(i S_{0}\right)$ gives the semiclassical representation for the Coulomb wave function $\phi^{(-)}(r, p ; w(\chi))$

$$
\phi^{(-)}(r, p ; w(\chi))=N_{0}(r) \exp \left(i S_{0}\right) .
$$

This single-particle wave function gives an effective description of propagation of the Wannier pair near the top of the Wannier ridge. The propagation is characterized by the momentum $p$ and the effective Coulomb charge $w(\chi)$. It is often more convenient to work directly with the Coulomb wave function rather than with its semiclassical representation (2.63), though one can keep open a possibility to return to the semiclassical description when necessary.

The quantity $\Sigma(r, \eta ; p, \chi)$ in (2.62) is defined by expansion (2.57), the coefficients $A(x), B(x), C(x)$ of which are given in (2.58), (2.51) and (2.52). From (2.62) we conclude that typical values for both $\chi$ and $\eta$ are restricted by the available above-threshold energy. For $\chi$ we find $\chi^{2} \sim p$. Similarly, for $\eta$ we find that $\eta^{2} \sim p \eta_{0}^{2}$. Here $\eta_{0}$ is a typical classical value of the momentum $\eta$ which according to (2.46) is estimated as $\eta_{0}=$ const $/ p$. For low energy, see (2.35), both $\chi$ and $\eta$ are small, $\chi^{2} \ll 1, \eta^{2} \ll \eta_{0}^{2}$. This fact justifies expansion (2.57) of the action in powers of $\chi$ and $\eta$.

Along with the wave function (2.62) one can consider another solution for the Wannier pair $\sim \sqrt{B} \exp (-i \Sigma) \phi^{(+)}$. We use conventional notation which shows explicitly 
the asymptotic behaviour of the Coulomb wave functions

$$
\phi_{p}^{( \pm)}(r ; w(\chi)) \rightarrow \frac{1}{\sqrt{p}} \exp \left[ \pm i\left(p r+\frac{w(\chi)}{p} \ln 2 p r\right)\right] .
$$

The normalization coefficient $1 / \sqrt{p}$ is convenient for the following calculations, see (5.2) in Section 5. At this stage an advantage of representation in which $\chi$ is a parameter and $\eta$ is an argument of wave functions becomes obvious. The potential $U(r, \chi)(2.4)$ approaches the value $-w(\chi) / r$ for $r \rightarrow \infty$ resulting in the effective Coulomb charge $w(\chi)$ which in this representation does not depend on arguments of the wave function and may be considered as a given constant. We have used this fact above to satisfy boundary conditions for large separation. In other representations, for example in representation in which $\chi$ is an argument of a wave function and $\eta$ is a parameter it is more laborious to satisfy these boundary conditions.

For effective use of the wave function (2.62) we will need to know the asymptotic form of the coefficients $A(x), B(x), C(x)$ in (2.57). It can be found from asymptotic conditions $(2.29),(2.30)$. Inside the Coulomb zone $\epsilon r \ll 1$, we find

$$
\begin{aligned}
& A(x) \simeq-w^{\prime \prime} d \\
& B(x) \simeq \zeta x^{-\nu}=\zeta\left(\frac{2 p^{2} r}{w}\right)^{\nu / 2} \\
& C(x) \simeq-\frac{1}{\nu+1} \frac{2}{w} x=-\frac{1}{\nu+1}\left(\frac{2}{w p^{2} r}\right)^{1 / 2} .
\end{aligned}
$$

Outside the Coulomb zone $\epsilon r \gg 1$ we find

$$
\begin{aligned}
& A(x) \simeq-\frac{2 w^{\prime \prime 2}}{p^{2} r} \rightarrow 0, \\
& B(x) \simeq 1, \\
& C(x) \simeq-\frac{1}{p^{2} r} \rightarrow 0 .
\end{aligned}
$$

Using (2.68),(2.69),(2.70) we verify that the phase in the definition of the wave function (2.62) is chosen in such a way that for $r \rightarrow \infty$

$$
\psi_{p, \chi}(r, \eta) \rightarrow \frac{1}{\sqrt{p}} \exp \left[i\left(\eta \chi-p r-\frac{w(\chi)}{p} \ln 2 p r\right)\right] .
$$

The discussion above shows that the main features of the Wannier problem are described by the effective single-particle Coulomb problem with the effective Coulomb charge $w(\chi)$. An essential property of the single-particle problem is a phase shift. In order to find it let us present it in the following form

$$
\delta(\chi)=\delta_{\mathrm{c}}(\chi)+\Delta \delta(\chi) .
$$


Here the first term is the usual Coulomb phase shift which arises due to the effective motion in the Coulomb field of the charge $w(\chi)$

$$
\delta_{\mathrm{c}}(\chi)=\arg \Gamma\left(1-i \frac{w(\chi)}{p}\right) .
$$

An additional phase shift $\Delta \delta(\chi)$ is produced by the term $\Sigma(r, \eta ; p, \chi)$ in the exponent in (2.62). Remember that this term is part of the classical action. The variation of the action when $r$ varies from $r=\infty$ to $r=0$ in the semiclassical approximation is identical to the phase shift. Therefore

$\Delta \delta(\chi)=-[\Sigma(r=\infty, \eta=0 ; p, \chi)-\Sigma(r=0, \eta=0 ; p, \chi)]=-\frac{w^{\prime \prime} d}{2 p} \chi^{2}$.

Evaluating the last equality (2.65),(2.68) were used. The minus sign in front of the square brackets in (2.74) agrees with definition (2.64) of the function $\phi_{p}^{(-)}(r ; w(\chi))$. From the asymptotic condition (2.71) we conclude that the found phases (2.73),(2.74) are the only ones which contribute to the phase shift (2.72).

The found representation for the wave function of the Wannier problem (2.62) has a clear physical meaning. The Wannier problem takes place in the vicinity of the Wannier ridge. This allows to present essential properties of the two-electron problem by the effective single-particle Coulomb problem. The Coulomb wave function $\phi_{p}^{(-)}(r ; w(\chi))$ of this effective Coulomb problem gives the simplest approximation. The nontrivial threebody nature of the Wannier problem is taken care of by the quantities $\Sigma(r, \eta ; p, \chi), B(x)$ which accounts for small fluctuations in the vicinity of the ridge-top trajectory. These fluctuations result in two interesting properties. Firstly, the state of the Wannier pair on the top of the ridge is unstable in respect to sliding down into configuration when one electron is close to the atom while another one is far apart. This reduces the probability for the pair to survive on the top of the ridge. This effect is taken into account by the normalization coefficient $B(x) \leq 1$. Secondly, there appears an additional important contribution to the phase shift $\Delta \delta(\chi)$ in the effective single-particle problem.

If the wave function (2.62) is used in the traditional Wannier problem to calculate the cross-section of a process with two Wannier electrons in the final state then the normalization coefficient $B(x)$ should be estimated with the help of (2.66) in the vicinity of the atom. This gives $|\psi| \sim \varepsilon^{\nu / 4}$, and therefore the threshold behaviour of the crosssection is estimated as $\sigma \sim|\psi|^{2} \sim \varepsilon^{\nu / 2}=\varepsilon^{\mu}$ reproducing the Wannier threshold law (1.2). 


\section{PCI in the near-threshold region}

The resonant nature of reaction (1.1), (1.4) permits one to present the matrix element describing it in the following general form

$$
M=\sum_{\mathrm{j}} \frac{\left\langle\Psi_{\mathrm{f}}\left|V_{\mathrm{A}}\right| \Psi_{\mathrm{j}}\right\rangle\left\langle\Psi_{\mathrm{j}}\left|V_{\mathrm{col}}\right| \Psi_{\mathrm{i}}\right\rangle}{\varepsilon-\varepsilon_{\alpha \mathrm{j}}-\varepsilon_{\beta \mathrm{j}}+i \Gamma / 2} .
$$

The wave function $\left|\Psi_{\mathrm{i}}\right\rangle$ describes the initial state $\mathrm{e}+\mathrm{A},\left|\Psi_{\mathrm{j}}\right\rangle$ describes the set of intermediate states $\mathrm{e}_{\alpha}+\mathrm{e}_{\beta}+\mathrm{A}^{+*}$ with the fixed resonant state of the ion $\mathrm{A}^{+*}$, and $\left|\Psi_{\mathrm{f}}\right\rangle$ describes the final state $\mathrm{e}_{\alpha}+\mathrm{e}_{\beta}+\mathrm{e}_{\gamma}+\mathrm{A}^{2+}$ of the reaction described by (1.1),(1.4). Summation in (3.1) takes into account all possible states for Wannier electrons $\mathrm{e}_{\alpha}, \mathrm{e}_{\beta}$ in the intermediate state. The potential $V_{\text {col }}$ describes the part of the Hamiltonian which is responsible for the collision, and $V_{\mathrm{A}}$ is the potential which causes the Auger decay. The energy denominator depends on the above-threshold energy as well as on the energies $\varepsilon_{\alpha \mathrm{j}}, \varepsilon_{\beta \mathrm{j}}$ of the Wannier electrons in the intermediate state and the total width $\Gamma$ of the autoionizing atomic state. We are interested in the low near-threshold energies for the Wannier pair (2.35). In contrast the autoionized electron is sufficiently fast and we can assume that its energy $\varepsilon_{\gamma}$ is so high that

$$
\varepsilon \ll 1 \ll \varepsilon_{\gamma} .
$$

The width $\Gamma$ is another small parameter of the problem. We will assume that the width is much lower than the above threshold energy

$$
\Gamma \ll \varepsilon \text {. }
$$

Inequalities (3.2), (3.3) greatly simplify the problem. Firstly, (3.3) implies that the decay takes place when the Wannier pair is far outside the atomic radius. As a result the electrons $\mathrm{e}_{\alpha}, \mathrm{e}_{\beta}$ have no influence on the process of ejecting the electron $\mathrm{e}_{\gamma}$. Secondly, inequality (3.2) guarantees that the interaction between the Wannier pair and the autoionized electron is small, and we can neglect it. As a result the matrix element $\left\langle\Psi_{\mathrm{f}}\left|V_{\mathrm{A}}\right| \Psi_{\mathrm{j}}\right\rangle$ reduces to

$$
\left\langle\Psi_{\mathrm{f}}\left|V_{\mathrm{A}}\right| \Psi_{\mathrm{j}}\right\rangle \simeq \sqrt{\Gamma_{\mathrm{f}}}\left\langle\psi_{2} \mid \psi_{\mathrm{j}}\right\rangle
$$

Here $\Gamma_{\mathrm{f}}$ is the partial width of the autoionizing state, which makes $\sqrt{\Gamma_{\mathrm{f}}}$ proportional to the matrix element responsible for the autoionization decay. It is determined by the processes which take place strictly inside the atomic particle and, as mentioned, are not influenced by PCI. The effects of PCI are taken care of in the overlapping integrals $\left\langle\psi_{2} \mid \psi_{\mathrm{j}}\right\rangle$ between the wave functions which describe the states of the Wannier pair. We denote by $\left\langle\psi_{2}\right|$ the final-state wave-function for the Wannier pair and by $\left|\psi_{\mathrm{j}}\right\rangle$ the full set of the intermediate-state wave functions for the pair. Substituting (3.4) in (3.1) we can present the matrix element $M$ in the following simple form

$$
M=\sqrt{\Gamma_{\mathrm{f}}}\left\langle\psi_{2} \mid \psi_{1}\right\rangle
$$


Here the wave function $\left|\psi_{1}\right\rangle$ is defined as

$$
\left|\psi_{1}\right\rangle=\left\langle\Psi\left(\mathrm{A}^{+*}\right)\left|G_{\varepsilon+i \Gamma / 2}^{(+)}\right| V_{\text {col }} \mid \Psi_{i}\right\rangle,
$$

where $\left\langle\Psi\left(\mathrm{A}^{+*}\right)\right|$ is the wave function of the resonant state of the ion. It projects the many-electron wave function $V_{\text {col }}\left|\Psi_{i}\right\rangle$ onto the states of the Wannier electrons. The function $\left|\psi_{1}\right\rangle$ depends on the coordinates of the two electrons. Summation over the full set of intermediate states for the pair is included in the two-particle Green function

$$
G_{\varepsilon+i \Gamma / 2}^{(+)} \equiv G_{\varepsilon+i \Gamma / 2}^{(+)}\left(\mathbf{r}_{1}, \mathbf{r}_{2} ; \mathbf{r}_{1}^{\prime}, \mathbf{r}_{2}^{\prime}\right)=\sum_{\mathrm{j}} \frac{\psi_{\mathrm{j}}\left(\mathbf{r}_{1}, \mathbf{r}_{2}\right) \psi_{\mathrm{j}}^{*}\left(\mathbf{r}_{1}^{\prime}, \mathbf{r}_{2}^{\prime}\right)}{\varepsilon-\varepsilon_{\alpha \mathrm{j}}-\varepsilon_{\beta \mathrm{j}}+i \Gamma / 2} .
$$

Equation (3.5) states that the influence of PCI on the Wannier pair can be described with the help of the overlapping integral. This result is very close in spirit to the shakedown model [13, 14] which originally was developed for the case when one near-threshold electron is exposed to the variation of the charge of the atomic particle caused by the autoionization. In the case under consideration there are two near-threshold electrons, but the idea that overlapping of the wave functions gives the correct description of PCI remains the same.

The wave functions in the matrix element (3.5) satisfy the Schroedinger equations

$$
\begin{aligned}
\left(\varepsilon+i \frac{\Gamma}{2}-H_{1}\right)\left|\psi_{1}\right\rangle & =|Q\rangle, \\
\left(E-H_{2}\right)\left|\psi_{2}\right\rangle & =0 .
\end{aligned}
$$

Here $H_{1}$ and $H_{2}$ are the Hamiltonians which describe propagation of the Wannier pair in the intermediate and final states

$$
H_{n}=-\frac{1}{2}\left(\Delta_{\alpha}+\Delta_{\beta}\right)+\frac{1}{r_{\alpha \beta}}-Z_{n}\left(\frac{1}{r_{\alpha}}+\frac{1}{r_{\beta}}\right) .
$$

An index $n=1,2$ here labels the intermediate and the final states in which the Coulomb charge of the atomic particle is different. Generally speaking there are also the short-range potentials which account for the potential of the atomic particle in the intermediate or final states. However, the influence of the short-range potential on PCI is small, and these potentials are neglected in (3.10) and the following consideration. The energy $\varepsilon+i \Gamma / 2$ of the pair in the state $\left|\psi_{1}\right\rangle$ is complex. Its real part $\varepsilon$ is equal to the above-threshold energy, its imaginary part $\Gamma / 2$ appears due to autoionization decay. The function $\left|\psi_{2}\right\rangle$ describes the Wannier pair in the final state with real energy $E$ which satisfies the energy conservation law $E_{\text {col }}+E(\mathrm{~A})=E+\varepsilon_{\gamma}+E\left(\mathrm{~A}^{2+}\right)$, where $E(\mathrm{~A})$ is the energy of the atom, $E\left(\mathrm{~A}^{2+}\right)$ is the energy of the ion, $\varepsilon_{\gamma}$ is the energy of the autoionized electron, and $E_{\text {col }}$ is the collision energy.

According to (3.6),(3.7) there appears a source in the right-hand side of the Schroedinger equation (3.8)

$$
|Q\rangle=\left\langle\Psi\left(\mathrm{A}^{+*}\right)\left|V_{\text {col }}\right| \Psi_{i}\right\rangle
$$


which is a function of the coordinates of the pair. For large separations from the ion this function becomes proportional to the amplitude of inelastic scattering and inversely proportional to separation. It is important that the effects of PCI take place far outside the atomic particle [9], where the source is small $\left\langle r_{\alpha}, r_{\beta} \mid Q\right\rangle \simeq 0, r_{\alpha}, r_{\beta} \gg 1$. Therefore it is sufficient for our purposes to solve only the homogeneous Schroedinger equation for the function $\left|\psi_{1}\right\rangle$

$$
\left(\varepsilon+i \frac{\Gamma}{2}-H_{1}\right)\left|\psi_{1}\right\rangle=0
$$

in the region far outside the atom and then normalize this function to make it proportional to the amplitude of inelastic scattering, thus taking into account the influence of the source.

The given consideration shows that PCI for the Wannier pair in the vicinity of the threshold is described by the overlapping integral of the wave functions describing the

pair in the intermediate and final states (3.5). Notice that this possibility follows from conditions (3.2),(3.3). In this sense it is rather a theoretical result then a model.

\section{PCI for the Wannier pair}

Let us calculate the overlapping integral (3.5) using the wave functions for the Wannier problem evaluated in Section 2. The model suggested in Section 2 describes the state of the Wannier pair by its energy and the quantum number $\chi$. The amplitude of the inelastic collision depends on these quantum numbers

$$
\mathcal{A}(\chi)=\left\langle\varepsilon, \chi ; \Psi\left(\mathrm{A}^{+*}\right)\left|V_{\mathrm{col}}\right| \Psi_{i}\right\rangle .
$$

The matrix element in the right-hand side describes the events which take place in the vicinity of the atom. The small width $\Gamma$ of the autoionizing state does not manifest itself for these small separations. That is why we can neglect it, supposing that the wave function $\langle\varepsilon, \chi|$ in (4.1) describes a real physical state of the pair with the real energy $\varepsilon$.

The solution of (3.12) can be presented in the following form

$$
\left|\psi_{1}\right\rangle=\int\left[\psi_{k, \chi^{\prime}}(r, \eta)\right]_{1} \mathcal{A}\left(\chi^{\prime}\right) d \chi^{\prime}
$$

Here the wave function $\psi_{k, \chi^{\prime}}(r, \eta)$ defined in (2.62) describes a state of the Wannier pair

$$
\left[\psi_{k, \chi^{\prime}}(r, \eta)\right]_{1}=\left[\sqrt{B(x)} \exp \left[-i \Sigma\left(r, \eta ; k, \chi^{\prime}\right)\right] \phi_{k}^{(+)}\left(r ; w\left(\chi^{\prime}\right)\right)\right]_{1} .
$$

The square brackets with the index 1 are used to remind that the Wannier pair is in the intermediate state in which the Coulomb charge of the ion is $Z=Z_{1}=1$. 
Asymptotic condition (2.71) guarantees that the coefficients $\mathcal{A}\left(\chi^{\prime}\right)$ in (4.2) are identical to the amplitude of inelastic scattering.

It is important that dependence of the amplitude $\mathcal{A}\left(\chi^{\prime}\right)$ on $\chi^{\prime}$ can be established explicitly. Remember that (2.62) expresses the properties of the Wannier problem in terms of the effective single-particle problem. This allows one to present the amplitude of the collision as the following product

$$
\mathcal{A}\left(\chi^{\prime}\right)=\exp \left[i \delta\left(\chi^{\prime}\right)\right] \mathcal{A}_{0}\left(\chi^{\prime}\right) \simeq \exp \left[i \delta\left(\chi^{\prime}\right)\right] \mathcal{A}_{0}(\chi) .
$$

Here $\delta\left(\chi^{\prime}\right)$ is the phase shift which was found in (2.72), (2.73), (2.74). The quantity $\mathcal{A}_{0}\left(\chi^{\prime}\right)$ is a matrix element of inelastic collision. Representation (4.4) has a form which is conventional in the processes with single-particle final states [11]. The matrix element $\mathcal{A}_{0}\left(\chi^{\prime}\right)$ originates from integration in the vicinity of the atom. In contrast, the phase shift $\delta(\chi)$ arises due to the events which take place far outside the atom in the Coulomb zone. Thus the two quantities $\mathcal{A}_{0}\left(\chi^{\prime}\right)$ and $\delta\left(\chi^{\prime}\right)$ originate from quite different distances. This makes them depend differently on $\chi^{\prime}$. The phase shift exhibits strong variation $\delta\left(\chi^{\prime}\right) \sim \chi^{\prime 2} / k$, while the amplitude $\mathcal{A}_{0}\left(\chi^{\prime}\right)$ varies smoothly $\mathcal{A}_{0}\left(\chi^{\prime}\right) \sim \chi^{\prime 2}$. In order to verify this statement one should remember that in the semiclassical region all quantities depend on the classical action $\sim \int d r \sqrt{k^{2}+w\left(\chi^{\prime}\right) / r}$. For large $r, r \gg k^{2}$, the action has a term $\sim \chi^{\prime 2} / k$ which strongly varies with $\chi^{\prime}$, while for small $r, r \ll k^{2}$, there is only a smooth behaviour $\sim \chi^{\prime 2} \ll 1$. More general verification of this property can be fulfilled using the Landau-Smorodinsky approach to the problem of a low-energy particle influenced by a long-range Coulomb field as well as a short-range potential. It is discussed in detail in [11.

The smooth behaviour of the matrix element $\mathcal{A}_{0}\left(\chi^{\prime}\right)$ with $\chi^{\prime}$ justifies the last equality in (4.4). Using it we simplify (4.2)

$$
\left|\psi_{1}\right\rangle=\mathcal{A}_{0}(\chi) \int\left[\exp \left[i \delta\left(\chi^{\prime}\right)\right] \psi_{k, \chi^{\prime}}(r, \eta)\right]_{1} d \chi^{\prime} .
$$

The momentum $k$ in the state $\left|\psi_{1}\right\rangle$ is a complex number due to the finite width of the autoionizing state

$$
k=\sqrt{2 \varepsilon+i \Gamma} \simeq \sqrt{2 \varepsilon}+i \frac{\Gamma}{2 \sqrt{2 \varepsilon}} .
$$

Inequality (3.3) permits one to make expansion in the last equality in (4.6). It is important to keep the width in this expansion because the wave function (4.2) will be used to calculate the effects of PCI which take place for large separations. The positive sign of the imaginary part of $k$ ensures that the wave function decreases exponentially $\left[\psi_{k, \chi}(r, \eta)\right]_{1} \sim \exp (-\operatorname{Im} k r)$ with separation. Thus the events which result in PCI are localized in the finite region. Similar localization of the wave function is well known [9] for the case when one electron is influenced by PCI. We see that localization in the 
vicinity of the atom

$$
r \leq \frac{1}{\operatorname{Im} k} \simeq \frac{2 \sqrt{2 \varepsilon}}{\Gamma} \equiv r_{1}
$$

remains true for the Wannier pair as well. The radius of localization $r_{1}$ has a clear physical meaning being proportional to classical distances $r_{1}(t) \simeq r_{2}(t) \simeq r_{1} /(2 \sqrt{2})$ which separate Wannier electrons from the atom at the moment $t=1 / \Gamma$ of the decay.

The wave function $\left\langle\psi_{2}\right|$ in the overlapping integral (3.5) describes the real final state of the Wannier pair with the real energy E. Generally speaking this state should be described by a linear combination of outgoing and incoming waves. For our purposes it is necessary to take into account the convergent wave because it gives the major contribution to the overlapping integral and therefore correctly describes PCI. Really, the convergent wave function behaves like $\sim \exp (-i p r), p=\sqrt{2 \varepsilon}$ therefore the overlapping integral with the divergent wave function $\sim \exp (i k r)$ can be roughly estimated as $\sim 1 /(k-p) \sim 1 / \Gamma$ describing the resonance. In contrast, the divergent wave does not contribute to the resonance. This shows that the final-state wave function $\left|\psi_{2}\right\rangle$ can be taken in the form (2.62)

$$
\left|\psi_{2}\right\rangle=\left[\psi_{p, \chi}(r, \eta)\right]_{2}=\left[\sqrt{B(x)} \exp [i \Sigma(r, \eta ; p, \chi)] \phi_{p}^{(-)}(r ; w(\chi))\right]_{2} .
$$

The square brackets with the index 2 are to remind that all the quantities in the square brackets should be calculated for the final state in which the Coulomb charge of the ion is $Z=Z_{2}=2$ and the momentum of the pair is $p=\sqrt{2 E}$. Notation introduced in (4.3), (4.8) will be used below as well. All quantities labeled by an index 1 are considered for the intermediate state of the Wannier pair. In this state the ion charge is $Z=Z_{1}=1$, the energy of the pair is $\varepsilon+i \Gamma / 2$, the corresponding momentum is $k$ (4.6). The quantities labeled by an index 2 describe the final state with $Z=Z_{2}=2$, the final-state energy of the pair $E$ and the corresponding momentum $p=\sqrt{2 E}$.

The explicit form of the wave functions (4.5, 4.8) permits to present the matrix element describing PCI (3.5) in the following final form

$$
M=\sqrt{\Gamma_{\mathrm{f}}} \mathcal{A}_{0}(\chi) S_{21},
$$

where

$$
S_{21}=\int\left[\psi_{p, \chi}(r, \eta)\right]_{2}\left[\psi_{k, \chi^{\prime}}(r, \eta)\right]_{1} \exp \left[i \delta_{1}\left(\chi^{\prime}\right)\right] \frac{d \chi^{\prime} d \eta d r}{2 \pi}
$$

The above given consideration shows that this representation has a simple physical meaning. The fast autoionization decay results in an abrupt transition of the Wannier pair from the intermediate state to the final state. This transition is described by the overlapping integral of the wave functions for the intermediate and final states

$$
\left\langle\psi_{p, \chi, 2} \mid \psi_{k, \chi^{\prime}, 1}\right\rangle=\int\left[\psi_{p, \chi}(r, \eta)\right]_{2}\left[\psi_{k, \chi^{\prime}}(r, \eta)\right]_{1} \frac{d \eta d r}{2 \pi}
$$


The integration over $d \chi^{\prime}$ in (4.10) reflects the fact that the intermediate state includes the coherent superposition of the states of the pair with different $\chi^{\prime}$ excited due to inelastic collision. The factor $\exp \left[i \delta_{1}\left(\chi^{\prime}\right)\right]$ describes fast variation of the amplitude of the excitation with $\chi^{\prime}$.

Integration in (4.10) over $d \eta$ is Gaussian, therefore it can be fulfilled analytically, allowing the presentation of the amplitude $S_{21}$ as a two-dimensional integral which can be easily handled numerically.

Knowing the matrix element $M$ one can present the cross-section of the reaction (1.1), (1.4) in the form

$$
d \sigma_{\mathrm{pci}}(E)=\frac{\Gamma_{\mathrm{f}}}{\Gamma} R_{\mathrm{pci}}(E, \varepsilon) d \sigma_{0}(\varepsilon) .
$$

The cross-section $d \sigma_{\mathrm{pci}}(E)$ describes the full reaction in which PCI plays a role. The cross-section $d \sigma_{0}(\varepsilon)$ describes only reaction (1.1) in which the role of PCI is neglected. The factor

$$
R_{\mathrm{pci}}(E, \varepsilon)=\frac{\Gamma}{2 \pi}\left|S_{21}\right|^{2}
$$

presents the influence of PCI in the explicit form described by the amplitude $S_{21}$ in (4.10). This factor has a resonant nature sharply depending both on the above-threshold energy $\varepsilon$ and the final-state energy of the pair $E$.

Equations (4.10), (4.12), 4.13) explicitly describe the influence of PCI on the crosssection. The physical consequences of these formulae are discussed in the next Section 5 .

\section{Manifestations of PCI}

\subsection{Lorentz line}

Consider first the trivial situation when one neglects the effects of PCI supposing that the Coulomb charge of the ion in the intermediate and final state remains the same $Z_{1}=Z_{2}$. Then the wave functions marked by symbols 1 and 2 in (4.2),(4.8) differ only by the momenta $k$ and $p$. Notice that the coefficients $A(x), B(x), C(x)$ in the expansion of $\Sigma(r, \eta ; p, \chi)$ (2.57) are smooth functions. Therefore using inequality (3.3) we can assume that they do not vary much when the energy parameter $\epsilon$ in (2.16) takes either the value $\epsilon=E$, or $\epsilon=\varepsilon$, i.e. $\left.\left.A(x)\right|_{\epsilon=E} \simeq A(x)\right|_{\epsilon=\varepsilon},\left.\left.B(x)\right|_{\epsilon=E} \simeq B(x)\right|_{\epsilon=\varepsilon},\left.\left.C(x)\right|_{\epsilon=E} \simeq C(x)\right|_{\epsilon=\varepsilon}$. The last equality greatly simplifies integration over $d \eta$ in (4.10)

$$
\int \exp \left[i B(x)\left(\chi-\chi^{\prime}\right) \eta\right] \frac{d \eta}{2 \pi}=\frac{1}{B(x)} \delta_{D}\left(\chi-\chi^{\prime}\right) .
$$

Here $\delta_{D}\left(\chi-\chi^{\prime}\right)$ is the Dirac delta-function, which is eliminated by the following integration over $d \chi^{\prime}$ in (4.10). As a result the amplitude can be presented as the simple 
one-dimensional integral

$$
S_{21}=\exp [i \delta(\chi)] \int_{0}^{\infty} \phi_{p}^{(-)}(r ; w(\chi)) \phi_{k}^{(+)}(r ; w(\chi)) d r
$$

with the Coulomb wave functions in the field of the same effective Coulomb charge $w(\chi)$. Evaluating it with the help of (2.64) and substituting into (4.13) one finds that the resonant factor $R_{\text {pci }}(E, \varepsilon)$ reduces to the Lorentz line $R_{\mathrm{ll}}(E, \varepsilon)$

$$
R_{\mathrm{pci}}(E, \varepsilon) \rightarrow R_{\mathrm{ll}}(E, \varepsilon) \equiv \frac{\Gamma / 2 \pi}{(E-\varepsilon)^{2}+\Gamma^{2} / 4},
$$

as it should be when PCI is neglected. Notice that this result justifies the normalization of the wave functions in (2.64).

\subsection{Eikonal region}

Let us examine now the influence of PCI on the Wannier pair. The integration over $d r$ in (4.10) is localized in the region $r \leq r_{1}$ (4.7). It is essential how the localization radius $r_{1}$ is related to the Coulomb radius $r_{\mathrm{c}}$ (2.34). Let us examine the situation when condition

$$
r_{1} \equiv \frac{2 \sqrt{2 \varepsilon}}{\Gamma} \gg r_{\mathrm{c}} \equiv \frac{1}{E}
$$

is fulfilled. It is valid for sufficiently high energies $E, \varepsilon$. In this case the main contribution to the integral (4.10) comes from the region where asymptotic relations (2.68),(2.69),(2.70) are fulfilled. They imply that $B_{1}(r, k) \simeq B_{2}(r, p) \simeq 1$ and $C_{1}(r, k) \simeq C_{2}(r, p) \simeq 0$. These equalities show that the integration over $d \eta d \chi^{\prime}$ in (4.10) gives the trivial result

$$
\int \exp \left[i\left(\chi-\chi^{\prime}\right) \eta\right] \frac{d \eta d \chi^{\prime}}{2 \pi}=1
$$

Using it we find that the matrix element $S_{21}$ is simplified to be proportional to a onedimensional overlapping integral

$$
\begin{aligned}
S_{21} & =\exp \left[i \delta_{1}(\chi)\right]\left\langle\phi_{2} \mid \phi_{1}\right\rangle, \\
\left\langle\phi_{2} \mid \phi_{1}\right\rangle & =\int_{0}^{\infty} \phi_{p}^{(-)}\left(r ; w_{2}(\chi)\right) \phi_{k}^{(+)}\left(r ; w_{1}(\chi)\right) d r .
\end{aligned}
$$

Both wave functions here describe the effective single-particle Coulomb problem. This problem in the intermediate state is governed by the effective charge $w_{1}(\chi)$ defined by (2.6), (2.7),(2.8) with the ion charge $Z=Z_{1}=1$. In the final state the effective charge $w_{2}(\chi)$ depends on the final-state ion charge $Z=Z_{2}=2$.

From (5.5), (4.13) we find the following simple representation for the resonant factor

$$
R_{\mathrm{pci}}(E, \varepsilon) \simeq R_{\mathrm{c}}(E, \varepsilon) \equiv \frac{\Gamma}{2 \pi}\left|\left\langle\phi_{2} \mid \phi_{1}\right\rangle\right|^{2}
$$


The factor $R_{\mathrm{c}}(E, \varepsilon)$ defined in (5.6) depends on the overlapping integral between the wave functions describing the effective single-particle Coulomb problem. It is remarkable that this integral proves to be similar to the quantity which was well-known previously in the traditional PCI problem when a single low-energy electron is influenced by the products of the autoionization decay. In this case the shake-down model [13, 14, 9] gives correct description of PCI. The shake-down model is based on an overlapping integral with single-electron wave functions, whose structure is very close to the integral in (5.6), (5.7).

We come to a very interesting conclusion. Our knowledge of PCI properties of the traditional situation when there is only one near-threshold electron enables us to predict manifestations of PCI when the Wannier pair is involved. Conveniently we do not even need to fulfill explicitly calculation of the overlapping integral (5.5) which appears in (5.7). Inequality (5.3) guarantees that after the decay the Wannier pair is located so far away from the atom that neither the Coulomb charge of the ion nor repulsion between the electrons can significantly change the classical trajectory. Thus the pair moves almost without acceleration, which greatly simplify behaviour of the wave functions describing this motion. A similar case was well studied in the singleelectron problem. It was shown [9] that the eikonal approximation and the shake-down model give the same results when conditions of applicability for the eikonal theory are fulfilled. Borrowing this result from the true single-electron problem and applying it to our effective single-particle problem we conclude that the eikonal theory proves to be correct for the Wannier pair if condition (5.3) is fulfilled. Notice that condition (5.3) can be rewritten in the standard in the eikonal approximation form $\varepsilon^{3 / 2} \gg \Gamma$. Of course one could anticipate from the very beginning that when this usual condition is valid, then the eikonal theory should give the correct description of PCI for the Wannier pair. However, the peculiarity of the Wannier problem certainly appeals for a steady basis for such a statement. That is why it is important that we have been able to demonstrate validity of the eikonal theory by direct calculations.

Recognizing that the eikonal approximation is valid, we conclude that PCI for the Wannier pair should have the following manifestations.

1. The line in the autoionization spectrum is shifted by PCI. The shift is equal to [15, 9]

$$
\begin{aligned}
\Delta E & =-\frac{\Gamma}{2} \xi, \\
\xi & =\frac{w_{2}-w_{1}}{\sqrt{2 \varepsilon}}=\frac{1}{\mathrm{v}_{\alpha}}+\frac{1}{\mathrm{v}_{\beta}} .
\end{aligned}
$$

Here $\mathrm{v}_{\alpha}=\mathrm{v}_{\beta}=\sqrt{\varepsilon}$. The right-hand side in (5.9) is presented in the form which is common in the eikonal approximation.

2. The line changes the shape, becoming broader, lower, and acquiring the "left shoulder" which makes it asymmetrical. All these variations are the more significant 
the larger is the basic parameter $\xi$. These properties of the line are described by the profile [15, 9]

$$
\begin{aligned}
& R_{\mathrm{pci}}(E, \varepsilon) \simeq R_{\mathrm{eik}}(E, \varepsilon)=\frac{\Gamma / 2 \pi}{(E-\varepsilon)^{2}+\Gamma^{2} / 4} k_{\mathrm{eik}}((E-\varepsilon) / \Gamma, \xi), \\
& k_{\mathrm{eik}}((E-\varepsilon) / \Gamma, \xi) \equiv \frac{\pi \xi}{\sinh \pi \xi} \exp \left(-2 \xi \arctan \frac{E-\varepsilon}{\Gamma / 2}\right) .
\end{aligned}
$$

3. In spite of strong variations in the shape and position the intensity of the line remains constant, it is not influenced by PCI,

$$
\int R_{\mathrm{pci}}(E, \varepsilon) d E=1 \text {. }
$$

This property is essential for the Wannier problem where intensity is one of the main characteristics of the process. Taking into account that the cross-section of the reaction (1.1) satisfies the power law (1.2) with the index $\mu=\mu_{1}=1.127$ we find from (1.2) (4.12), (5.11) that this law remains valid for the total intensity of the line for reaction $(1.1),(1.4)$

$$
\sigma=\int \frac{d \sigma_{\mathrm{pci}}(E)}{d E} d E \sim \sigma_{0} \sim \varepsilon^{\mu_{1}}
$$

considered as a function of the above-threshold energy.

Up to this point our consideration was restricted by two particular assumptions. Firstly, we considered the case of large velocities of the autoionized electron (3.2) which allows one to assume that it does not play a role in PCI. Secondly, we discussed the opposite directions for the Wannier electrons neglecting fluctuations in their angular distribution. Once we have established that the eikonal theory is correct we can remove both these simplifications and include into our scheme the case of sufficiently low-energy autoionization and arbitrary directions for the Wannier electrons. As usual in the eikonal approximation it is sufficient to redefine the parameter $\xi$ which has the following more general form

$$
\xi=\frac{1}{\mathrm{v}_{\alpha}}+\frac{1}{\mathrm{v}_{\beta}}-\frac{1}{\mathrm{v}_{\alpha \gamma}}-\frac{1}{\mathrm{v}_{\beta \gamma}} .
$$

Here $\mathbf{v}_{\alpha \gamma}=\mathbf{v}_{\alpha}-\mathbf{v}_{\gamma}, \mathbf{v}_{\beta \gamma}=\mathbf{v}_{\beta}-\mathbf{v}_{\gamma}$. With this parameter $\xi$ (5.8),(5.10) describe the line which depends on the absolute values as well as directions of the velocities of all three electrons.

\subsection{Coulomb region}

Consider the case when the localization radius $r_{1}$ (4.7) is smaller than the Coulomb radius $r_{\mathrm{c}}$ (2.34)

$$
r_{\mathrm{l}} \equiv \frac{2 \sqrt{2 \varepsilon}}{\Gamma} \ll r_{\mathrm{c}} \equiv \frac{1}{E} .
$$


This inequality holds for sufficiently low energies $E, \varepsilon$. This inequality means that the decay takes place when the pair is so close to the ion that the Coulomb potential has a strong influence on trajectories of the Wannier pair.

Let us examine the behaviour of the integrand in the amplitude $S_{21}$ in (4.10) as a function of $\chi^{\prime}$. Remember that in the region $r \ll r_{\mathrm{c}}$ the asymptotic relations (2.65),(2.66), and (2.67) for the coefficients governing the action $\Sigma$ are valid. Notice first of all that the quadratic in $\chi^{\prime}$ terms in the exponent arising from the phase shift $\Delta \delta_{1}\left(\chi^{\prime}\right)$ and from the term $\left[\Sigma\left(r, \eta ; p, \chi^{\prime}\right)\right]_{1}$ cancel each other due to $(2.65),(2.74)$. Another simplification comes from the fact that for small distances the Coulomb function smoothly depends on $\chi^{\prime}$ exhibiting behaviour $\sim \chi^{\prime 2}$, see discussion after (4.4). Therefore one can suppose that

$$
\left[\exp \left[i \delta_{\mathrm{c}}\left(\chi^{\prime}\right)\right] \phi_{k}^{(+)}\left(r ; w\left(\chi^{\prime}\right)\right)\right]_{1} \simeq\left[\exp \left[i \delta_{\mathrm{c}}(\chi)\right] \phi_{k}^{(+)}(r ; w(\chi))\right]_{1} .
$$

These properties of the integrand allow analytical integration over $d \chi^{\prime}$ in (4.10)

$$
\int \exp \left(-i[B(x)]_{1} \chi^{\prime} \eta\right) \frac{d \chi^{\prime}}{2 \pi}=\frac{1}{[B(x)]_{1}} \delta_{D}(\eta)
$$

The delta-function is eliminated by the subsequent integration over $d \eta$. As a result one finds the following simple representation for the amplitude

$$
S_{21}=\exp \left[i \delta_{c, 1}(\chi)\right]\left\langle\phi_{2}\left|\left(B_{2} / B_{1}\right)^{1 / 2}\right| \phi_{1}\right\rangle .
$$

Here the matrix element is defined as

$$
\left\langle\phi_{2}\left|\left(B_{2} / B_{1}\right)^{1 / 2}\right| \phi_{1}\right\rangle=\int_{0}^{\infty} \phi_{p}^{(-)}\left(r ; W_{2}(\chi)\right)\left([B(x)]_{2} /[B(x)]_{1}\right)^{1 / 2} \phi_{k}^{(-)}\left(r ; W_{1}(\chi)\right) d r .
$$

Using (5.16) we find the following representation for the resonant factor (4.13)

$$
R_{\mathrm{pci}}(E, \varepsilon)=\frac{\Gamma}{2 \pi}\left|\left\langle\phi_{2}\left|\sqrt{B_{2} / B_{1}}\right| \phi_{1}\right\rangle\right|^{2} .
$$

This expression can be simplified even further. The functions $[B(x)]_{1}$ and $[B(x)]_{2}$ smoothly depend on the coordinate $r$. In contrast, the Coulomb wave functions $\left|\phi_{1}\right\rangle,\left|\phi_{2}\right\rangle$ oscillate. Calculating the integral with sufficiently fast oscillating functions one can use the saddle-point method. The semiclassical phases of the wave functions $\left|\phi_{1}\right\rangle,\left|\phi_{2}\right\rangle$ are $\int \sqrt{2\left(\varepsilon+i \Gamma / 2+W_{1}(\chi) / r\right)} d r$ and $\int \sqrt{2\left(E+W_{2}(\chi) / r\right)} d r$ respectively. Their difference is

$$
\Phi(r)=\int^{r}\left[\sqrt{2\left(\varepsilon+i \Gamma / 2+W_{1}(\chi) / r\right)}-\sqrt{2\left(E+W_{2}(\chi) / r\right)}\right] d r
$$

The saddle point $r=r_{\mathrm{sp}}$ is to satisfy equation $\Phi^{\prime}\left(r_{\mathrm{sp}}\right)=0$ yielding

$$
E+\frac{W_{2}(\chi)}{r_{\mathrm{sp}}}=\varepsilon+i \frac{\Gamma}{2}+\frac{W_{1}(\chi)}{r_{\mathrm{sp}}} .
$$


Solving this equation one finds

$$
r_{\mathrm{sp}}=\frac{W_{2}(\chi)-W_{1}(\chi)}{\varepsilon-E+i \Gamma / 2} .
$$

The energies $E, \varepsilon$ are low (5.14), therefore the radius $r=r_{\mathrm{sp}}$ is large. This fact justifies both the applicability of the semiclassical estimation for the phases in (5.19) and the validity of the saddle-point approximation. These arguments demonstrate that calculating the matrix element (5.17) we can suppose that the main contribution to the integral comes from the vicinity of the saddle point (5.21). This allows one to evaluate smooth functions $[B(x)]_{1},[B(x)]_{2}$ at this point and take them out of integration. As a result the resonant factor given by (5.18) can be simplified to the following final form which was first announced in (1.5) as the major result of this paper

$$
R_{\mathrm{pci}}(E, \varepsilon)=K_{\mathrm{w}}(E, \varepsilon) R_{\mathrm{c}}(E, \varepsilon),
$$

where the factor

$$
R_{\mathrm{c}}(E, \varepsilon) \equiv \frac{\Gamma}{2 \pi}\left|\left\langle\phi_{2} \mid \phi_{1}\right\rangle\right|^{2}
$$

depends on the overlapping integral $\left\langle\phi_{2} \mid \phi_{1}\right\rangle$. Notice that this integral can be presented in an analytical form if the integration is pushed one step further using the saddle-point method, but for our purposes it is sufficient to keep it in general form.

We met the factor (5.23) before when discussed the eikonal approximation, see (5.7). It was argued there that the factor $R_{\mathrm{c}}(E, \varepsilon)$ describes those properties of PCI which were previously well known from traditional studies of PCI with one low-energy electron. In contrast, the factor

$$
K_{\mathrm{w}}(E, \varepsilon)=\left|\frac{B_{2}\left(x_{2, s p}\right)}{B_{1}\left(x_{1, s p}\right)}\right|
$$

which appears in (5.22) is a new quantity specific for PCI with Wannier electrons, having no analogue in PCI with one low-energy electron. Due to this reason it is called the Wannier factor. Remember that the function $B(x)$ defined by $(2.51),(2.26),(2.16)$ gives normalization for the wave function of Wannier electrons (2.62). The found Wannier factor depends on a ratio of these normalization functions for the intermediate and final states. The coordinates of normalization functions, in accordance with (2.16) are defined as

$$
\begin{aligned}
& x_{1, s p}=\frac{1}{2}\left[\left(1+\frac{W_{1}(\chi)}{\varepsilon r_{\mathrm{sp}}}\right)^{1 / 2}+1\right], \\
& x_{2, s p}=\frac{1}{2}\left[\left(1+\frac{W_{2}(\chi)}{E r_{\mathrm{sp}}}\right)^{1 / 2}+1\right] .
\end{aligned}
$$

Traditionally the interest in the Wannier problem has been inspired by the nearthreshold power-law of the cross-section (1.2). It originates from the asymptotic 
behaviour of the normalization coefficient $B(x)$ for small separations when $E r \ll 1$, see (2.66). Thus traditional studies of the Wannier problem can be considered as a probing of the normalization coefficient $B(x)$ in the particular asymptotic region well inside the Coulomb zone. PCI opens an interesting opportunity to investigate this coefficient in a much wider area, not necessarily restricted by the Coulomb zone. According to (5.25),(5.26) variation of the above-threshold energy $\varepsilon$ and the final-state energy $E$ leads to variation of $x_{1, s p}, x_{2, s p}$ in a broad area resulting in significant variation of normalization coefficients $[B(x)]_{1}$ and $[B(x)]_{2}$.

The result obtained (5.22) permits one to address the question of the threshold behaviour of the cross-section which is of particular interest for the Wannier problem. When the energy of the Wannier pair is low $E \rightarrow 0$ then one finds from (5.21),(5.26) that $r_{\mathrm{sp}} \simeq\left(W_{2}(\chi)-W_{1}(\chi)\right) / \varepsilon$ and $x_{2, s p} \sim 1 / \sqrt{E} \rightarrow \infty$. The later condition allows the use of asymptotic relation (2.66) which shows that $B_{2}\left(x_{2, s p}\right) \sim E^{\mu_{2}}, \quad \mu_{2}=\nu_{2} / 2$. From (5.24) one finds the same estimate for the Wannier factor

$$
R_{\mathrm{pci}}(E, \varepsilon) \sim E^{\mu_{2}} .
$$

Equation (5.22) gives the same estimate for the resonant factor $R_{\text {pci }}(E, \varepsilon) \sim E^{\mu_{2}}$ resulting in the threshold law for the differential cross-section as a function of the energy of the pair $E$

$$
\left(\frac{d \sigma}{d E}\right)_{p c i} \sim E^{\mu_{2}}, \quad E \rightarrow 0 .
$$

Similarly one can estimate the behaviour of the resonant factor on the above-threshold energy $R_{\text {pci }}(E, \varepsilon) \sim \varepsilon^{-\mu_{1}}, \quad \mu_{1}=\nu_{1} / 2$. Remembering that the cross-section $\sigma_{0}$ of the exhibits the usual Wannier power-type behaviour $\sigma_{0} \sim \varepsilon^{\mu_{1}}, \quad \varepsilon \rightarrow 0$ as a function of the above-threshold energy we find that the cross-section for the combined process (1.1),(1.4) does not depend on the above-threshold energy $\varepsilon$

$$
\left(\frac{d \sigma}{d E}\right)_{p c i} \sim \text { const }, \quad \varepsilon \rightarrow 0 .
$$

We see that PCI has a dramatic effect on the threshold behaviour of the cross-section. Firstly, the PCI results in the variation of the exponent. The exponent in (5.28) corresponds to the final-state charge of the ion $Z=Z_{2}=2$ which gives $\mu_{2}=1.056$. If the Auger decay is impossible, then the cross-section of the reaction (1.1) is described by the usual Wannier power law (1.2) which corresponds to the charge of the ion $Z=Z_{1}=1$ resulting in $\mu_{1}=1.127$. Secondly, the cross-section does not depend on the abovethreshold energy $\varepsilon$. These results agree qualitatively with the simple physical picture. If above threshold energy is low then the decay takes place when the pair is so close to the ion that its separation can be neglected compared with the large radius of the Coulomb zone. This means that the propagation of the pair in the intermediate state 
is insignificant, which makes insignificant as well the very existence of this intermediate state. That is why the final answer for the Wannier exponent should not depend on parameters governing the intermediate state. The fact that (5.22) reproduces this result can be considered as a qualitative verification of this formula.

Generally speaking, one could contemplate a possibility to measure the power-type behaviour (5.28). To this end one should fix the above-threshold energy and measure the "left shoulder" of the resonance profile in the near-threshold region versus the energy of the Wannier pair. However this project meets a difficulty. It can be verified that in order to distinguish the exponent $\mu_{2}$ from $\mu_{1}$ in the power-type behaviour of the cross-section (5.28) the left-hand side in inequality (5.14) should be really small, say, less than $\leq 10^{-2}$ putting a severe restriction on $E, \varepsilon$.

The most important result of this Subsection is (5.22) which describes the influence of PCI on the resonance profile. This equation was evaluated assuming that energies $E, \varepsilon$ are low enough to satisfy condition (5.14). Notice, however, that the equation remains correct for much higher energies satisfying the condition of applicability of the eikonal approximation (5.3) which is opposite to the low-energy limit. To see this consider the eikonal region (5.3) in which $E \sim \varepsilon \sim|E-\varepsilon| \gg \Gamma^{2 / 3}$. For these energies (5.21) results in $r_{\mathrm{sp}} \sim \Gamma^{-2 / 3}$ and therefore $E r_{\mathrm{sp}} \sim \varepsilon r_{\mathrm{sp}} \gg 1$. Equations (5.25),(5.26) show that $x_{1, s p} \simeq x_{2, s p} \simeq 1$ permitting one to use asymptotic condition (2.69) which shows that the Wannier factor is trivial $K_{\mathrm{w}}(E, \varepsilon) \simeq 1$. Therefore, in the eikonal region (5.22) yields $R_{\mathrm{pci}}(E, \varepsilon) \simeq R_{\mathrm{c}}(E, \varepsilon)$ which according to (5.7) is the correct result. The fact that (5.22) proves to be correct in both the eikonal region $r_{1} \ll r_{\mathrm{c}}$ and the Coulomb region $r_{1} \gg r_{\mathrm{c}}$ may be considered as an indication that it should give reasonable results in the intermediate region $r_{1} \sim r_{\mathrm{c}}$ as well.

\section{Electron impact in the vicinity of $\mathrm{Ne} \mathrm{K}$-shell}

In order to illustrate the validity of different approximations developed above for description of PCI let us consider the example studied experimentally in [1] in which the electron impact ionization of the Ne atom in the vicinity of the K-shell

$$
\mathrm{e}+\mathrm{Ne} \rightarrow \mathrm{e}+\mathrm{e}+\mathrm{Ne}^{+}\left(1 s^{-1}\right)
$$

is followed by the KLL Auger decay

$$
\mathrm{Ne}^{+}\left(1 s^{-1}\right) \rightarrow \mathrm{e}+\mathrm{Ne}^{2+}\left(2 p^{-2}\right)
$$

The distortion of resonance profiles by PCI is illustrated in figure 1 for abovethreshold energies $\varepsilon=2,3,5$ and $10 \mathrm{eV}$. The width of the $\mathrm{K}$-vacancy is $\Gamma=270 \mathrm{meV}$ [16]. The profiles are calculated for the symmetric geometry of Wannier electrons when they move in opposite directions possessing equal energies. Notice that experimentally 
[1] the spectra were measured versus the energy of the Auger electron when neither energy nor angular distributions of Wannier electrons were fixed.

The full curve in figure 1 presents the results of calculations based on the most advanced equation (5.22) which, as was argued above is valid for both near-threshold and high energy regions. The factor $R_{\mathrm{c}}(E, \varepsilon)=\Gamma /(2 \pi)\left|\left\langle\phi_{2} \mid \phi_{1}\right\rangle\right|^{2}$ is calculated by direct numerical integration of the overlapping integral with the Coulomb wave functions describing the effective single-particle problem. Remember that this problem gives the simplest description of the Wannier pair. Normalization of the Coulomb wave functions is given in (2.64). For numerical calculations it is more convenient to work with the real Coulomb wave function $\phi_{2}(r)$ taking a linear combination of convergent and divergent waves, rather then only the convergent wave. This is possible because the divergent wave in $\phi_{2}(r)$ does not contribute to the resonance, see discussion before (4.8). The wave function $\phi_{1}(r)$ has to be taken as a divergent wave localized in the vicinity of the atom [9]. The Wannier factor $K_{\mathrm{w}}(E, \varepsilon)$ is found from $(2.51),(2.26)$.

Full dotes in figure 1 present calculation based on (5.18). The perfect agreement between the full lines and the full circles shows the high accuracy of the approximation which was used in order to present the resonant factor in the final form (5.22) as a product of two different factors.

Dotted lines in figure 1 present the simple eikonal approximation (5.10) which should be correct only for high energies. Results presented show that below $\varepsilon=5 \mathrm{eV}$ the eikonal approximation fails, while for high above-threshold energies $\varepsilon \geq 10 \mathrm{eV}$ it produces quite reliable results. Notice that experimental results of [1] belong to the region $\varepsilon \geq 10 \mathrm{eV}$ where we have just verified the eikonal approximation to be valid.

The dotted line in figure 1( $a)$ shows the Coulomb factor $R_{\mathrm{c}}(E, \varepsilon)$ which is known to produce good results for PCI with a single low-energy electron. Figure $(1 a)$ shows that for the considered situation with two low-energy electrons it proves to be incorrect for low energies. This demonstrates that the Wannier factor $K_{\mathrm{w}}(E, \varepsilon)$ plays a very important role. The Wannier factor exhibits quite an interesting variation as demonstrated in figure 2 where it is presented versus the energy of the pair $E$ for the fixed above-threshold energy $\varepsilon=2 \mathrm{eV}$. In order to reveal the asymptotic low-energy behaviour $\sim E^{\mu_{2}}, E \rightarrow 0$ predicted by (5.28) the Wannier factor is shown as $K_{\mathrm{w}}(E, \varepsilon) / E$.

The PCI shift found from calculations of the PCI profiles is shown in figure 3 for the symmetrical configuration of Wannier electrons. The full curve gives the results obtained from (5.22). The dotted line shows the prediction of the eikonal approximation. The full dottes in figure 3 present the results extracted from the profiles calculated in [2]. These results effectively include different possible configurations of Wannier electrons in the final state. One can expect that the symmetrical configuration of the Wannier electrons gives large contribution to the total probability. Therefore the results calculated for the symmetrical configuration should reasonably agree with calculations in which all 
continuum states of Wannier electrons are included. Notice that the theory developed above and the approach of [2] are very different in technique used. Nevertheless there is a clear resemblance in several important basic features. Firstly, in both works PCI is described with the help of overlapping integrals between wave functions describing the Wannier pair. Secondly, both works rely upon the semiclassical approximation. Having in mind these basic similarities one could expect that the two approaches should give similar results. This hope proves be correct for high energies, see figure $1(d)$ for 10 $\mathrm{eV}$ above the threshold. There is, however, some discrepancy below $10 \mathrm{eV}$. It can be verified comparing figure 1 with the results presented in figure of [2] that distinctions exist in profile shapes as well.

\section{Discussion of the main results}

This paper develops the following ideas and results.

1.It is emphasized that the propagation of two Wannier electrons can be described by an effective single-particle Coulomb problem with corrections which take into account more subtle three-body properties. Correspondingly the wave function of the Wannier pair (2.62) is presented as the product of the Coulomb wave function describing the effective single-particle Coulomb problem, the additional phase factor $\exp (i \Sigma)$, and the normalization coefficient $B(2.51)$ which plays a very important role in our consideration.

2.The complex problem (1.1), (1.4) with three electrons in the final state is reduced to a more simple problem in which only two Wannier electrons play a role. The key point is an assumption that the autoionization energy is so high that the autoionized electron cannot interact strongly with the other two electrons. The dynamical properties responsible for PCI are shown to be described by the overlapping integral (4.10) between the wave functions of Wannier electrons in the final and intermediate states.

3. The resonant factor is found in a very simple form (5.22) which depends on two factors. One of them $R_{\mathrm{c}}$ describes the overlapping integral between the Coulomb wave functions for the effective single-particle problem. A similar integral is known to describe PCI when there is only one near-threshold electron. More specific properties of the considered problem are described by the Wannier factor $K_{\mathrm{w}}$. The factorization of the resonant factor into a product of $K_{\mathrm{w}}$ and $R_{\mathrm{c}}$ has a clear physical reason. There are two scales in the problem. The effective single-particle Coulomb problem describes strong variations of the wave function with a typical radius $r_{\text {single }} \sim 1 / p$. In contrast, the quantities $\Sigma, B$ describing the specific properties of the three-body problem vary with a typical distance equal to the radius of the Coulomb zone $r_{3 \text {-body }} \sim 1 / p^{2}$. For low energies these radii are different $r_{\text {single }} \ll r_{3 \text {-body }}$. It is well known that when there are two different scales in a problem then one has to expect an amplitude and a probability to be presented as products of quantities describing events which happen in different 
scales, as it happens in our case.

Equation (5.22) was verified for low energies, i.e. in the Coulomb region, and for high energies in the eikonal zone. For intermediate region one can hope that it should give reasonable results. (However, validity of the later assumption should be clarified by numerical integration of the matrix element (4.10).)

The Wannier factor $K_{\mathrm{w}}$ is shown to depend on the normalization coefficient $B$ of the wave function of the Wannier pair, see (5.24). The distance at which this coefficient is to be taken depends on the above-threshold energy as well as on the energy of the pair in the final state, see (5.21). It is remarkable that the same coefficient considered for small separations (2.66) governs the Wannier power law (1.2), and therefore it has been under thorough experimental investigation for a long period of time. Equation (5.22) shows that there is a new possibility to experimentally investigate the coefficient $B$ in a wide range of distances.

4.It is demonstrated that for low energies of the pair $E \rightarrow 0$ the Wannier power law has the form (5.27) with the exponent governed by the ion charge in the final state. In contrast for higher energies in the eikonal region the power law has a form of (5.12) depending on the above-threshold energy $\varepsilon$ with the exponent governed by the ion charge in the intermediate state.

5.Our consideration was restricted by the one-dimensional model formulated in Section 2. However, one can hope that the approach developed is more general than the model itself. There are clear physical reasons for this. Firstly, PCI as well as the Wannier problem manifest themselves for large separations where the Coulomb potential play an important role, while the more rapidly decreasing orbital barrier does not. Therefore neglect of this later potential seems reasonable. Secondly, the arguments considered above show that the factorization of the resonant factor into the product of the Coulomb factor and the Wannier factor is a general property caused by existence

of the two different scales in the problem. Therefore it should remain valid when the angular variables are included as well.

\section{Acknowledgments}

I am thankful to V.N.Ostrovsky who drawn my attention to the problem discussed in this paper providing me with the preprint of the paper [2]. The support of the Australian Research Council is acknowledged.

\section{References}

[1] Kamm M, Weber W and Mehlhorn W 1994 J.Phys.B:At.Mol.Opt.Phys. 272585 
[2] Kazansky A K and Ostrovsky V N 1996 5-th International Workshop "Autoionization Phenomena in Atoms", Eds.V.V.Balashov, A.A. Grum-Grzhimailo, and E.A.Romanovsky (Moscow) (Moscow University Press) p 67

[3] Wannier G H 1953 Phys.Rev. 90817

[4] Peterkop R 1971 J.Phys.B:At.Mol.Opt.Phys. 4513

[5] Rau A R P 1971 Phys.Rev.A 4207

[6] Klar H and Schlecht W 1976 J.Phys.B:At.Mol.Opt.Phys. 91699

[7] Watanabe S 1987 Phys.Rev.A 361566

[8] Kazansky A K and Ostrovsky V N 1992 J.Phys.B:At.Mol.Opt.Phys 252121

[9] Kuchiev M Yu and Sheinerman S A 1989 Sov.Phys.Uspechi 32569

[10] Peterkop R and Liepinsh 1969 Abstr. 6th Int. Conf on the Physics of Electronic and Atomic Collisions (Cambridge, Mass: M.I.T. Press) 212

[11] Landau L D and Lifshits E M 1977 Quantum mechanics 3d ed., rev. and enl. Course of theoretical physics; v.3. ( Oxford ; New York : Pergamon Press)

[12] Fock V A 1978 Fundamentals of quantum mechanics (Moscow : Mir)

[13] Read F 1975 Rad.Res. 6423

[14] King G C , Read F H and Bradford R C 1975 J.Phys.B:At.Mol.Opt.Phys 82210

[15] Kuchiev M Yu and Sheinerman S A 1986 Sov.Phys.-JETP 63986

[16] Krause M O and Oliver J H 1979 J.Chem.Phys.Ref.Data 8329 


\section{Figure captions}

Figure 1. Electron impact with excitation of K-shell in Ne. Calculated profiles of Auger lines distorted by PCI $R(E, \varepsilon)$ versus the energy of the Wannier pair $E$ for a fixed above-threshold energy $\varepsilon$. The width of the K-vacancy is $270 \mathrm{meV}$ [16]. Figures $(a),(b),(c)$, and $(d)$ present results for $\varepsilon=2,3,5$ and $10 \mathrm{eV}$. The symmetric configuration of Wannier electrons is considered, they move in opposite directions possessing equal energies. - - - - the non-distorted Lorentz line, _— prediction of the most advanced equation (5.22), — — the eikonal approximation $R_{\mathrm{eik}}(E, \varepsilon)(5.10)$, $\bullet$ prediction of equation (5.18), - - - the Coulomb factor $R_{\mathrm{c}}(E, \varepsilon)$ (5.23). The perfect agreement between the full line and full-circled one shows the high accuracy of the approximation which permits to present the resonant factor in the final form (5.22). Figure $(d)$ shows that the eikonal approximation is valid above $10 \mathrm{eV}$. The strong deviation of the dashed line from the full one in figure $(a)$ indicates an important role played by the Wannier factor $K_{\mathrm{w}}(E, \varepsilon)$ for low energies.

Figure 2. The same reaction as in figure 1. the ratio of the Wannier factor $K_{\mathrm{w}}(E, \varepsilon)$ to the energy of the pair $E$ versus the energy $E$ for the fixed above-threshold energy $\varepsilon=2 \mathrm{eV},---$ low-energy asymptotic behaviour (5.28).

Figure 3. The same reaction as in figure 1. The calculated PCI shift versus the above-threshold energy: — prediction based on equation (5.22), ---the eikonal approximation (5.10), ๑results extracted from profiles calculated in [2]. 


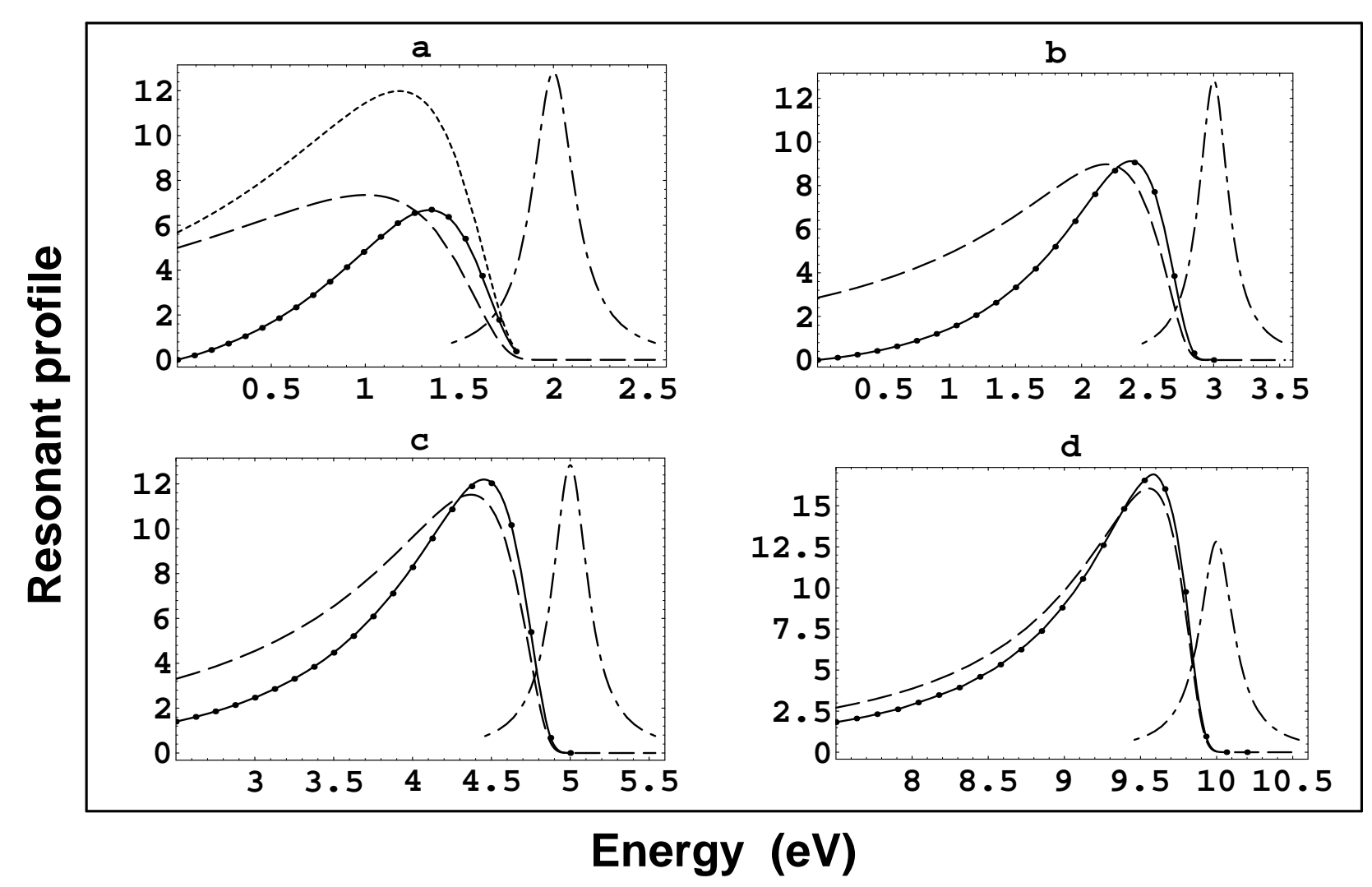

Figure 1 


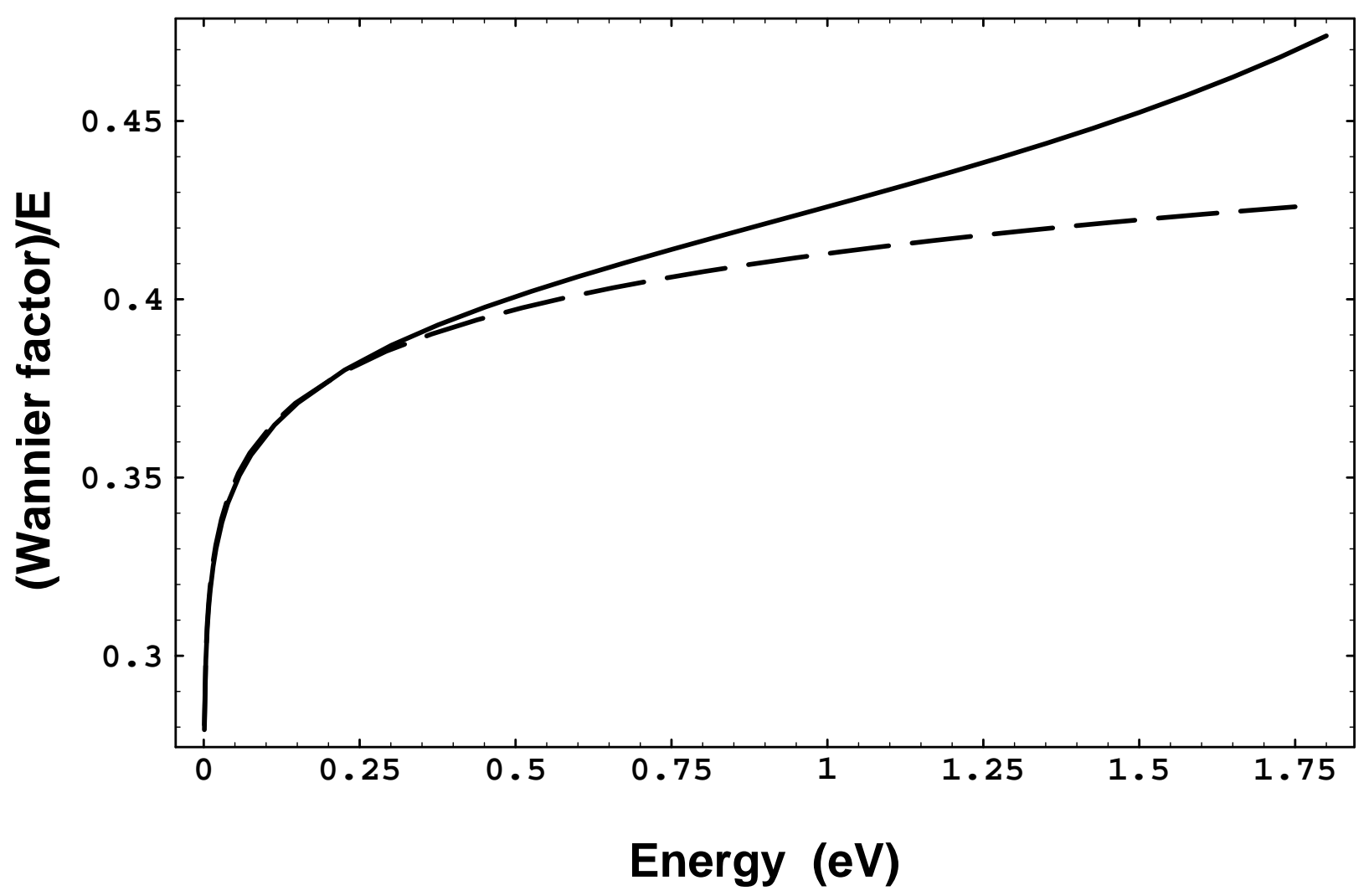

Figure 2 


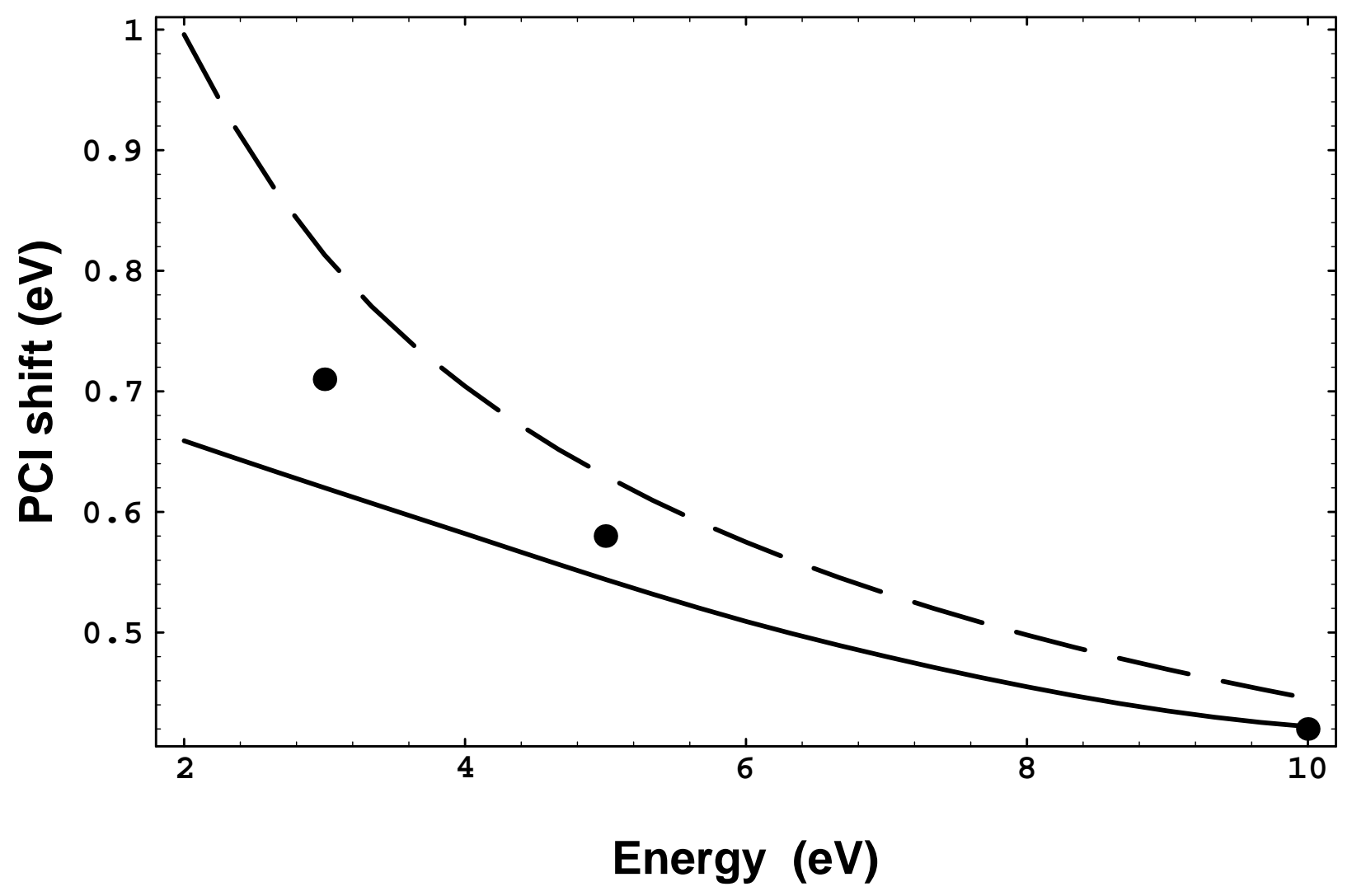

Figure 3 